\title{
On Massive MIMO Base Stations with Low-End Hardware
}

Christopher Mollén

\section{COMMUNICATION SîsTems}

Division of Communication Systems Department of Electrical Engineering (ISY) Linköping University, 58183 Linköping, Sweden www.commsys.isy.liu.se

Linköping 2016 
This is a Swedish Licentiate Thesis.

The Licentiate degree comprises 120 ECTS credits of postgraduate studies.

On Massive MIMO Base Stations with Low-End Hardware

(C) 2016 Christopher Mollén, unless otherwise noted.

ISBN 978-91-7685-707-6

ISSN 0280-7971

Printed in Sweden by LiU-Tryck, Linköping 2016 


\section{Abstract}

Massive Mimo (Multiple-Input Multiple-Output) base stations have proven, both in theory and in practice, to possess many of the qualities that future wireless communication systems will require. They can provide equally high data rates throughout their coverage area and can concurrently serve multiple low-end handsets without requiring wider spectrum, denser base station deployment or significantly more power than current base stations. The main challenge of massive MIMO is the immense hardware complexity and cost of the base station-each element in the large antenna array needs to be individually controllable and therefore requires its own radio chain. To make massive MIMO commercially viable, the base station has to be built from inexpensive simple hardware. In this thesis, it is investigated how the use of low-end power amplifiers and analog-to-digital converters (ADCs) affects the performance of massive MIMO. In the study of the signal distortion from low-end amplifiers, it is shown that in-band distortion is negligible in massive MIMO and that out-of-band radiation is the limiting factor that decides what power efficiency the amplifiers can be operated at. A precoder that produces transmit signals for the downlink with constant envelope in continuous time is presented to allow for highly power efficient low-end amplifiers. Further, it is found that the out-of-band radiation is isotropic when the channel is frequency selective and when multiple users are served; and that it can be beamformed when the channel is frequency flat and when few users are served. Since a massive MIMO base station radiates less power than today's base stations, isotropic out-of-band radiation means that low-end hardware with poorer linearity than required today can be used in massive MIMO. It is also shown that using one-bit ADCs-the simplest and least power-hungry ADCs - at the base station only degrades the signal-to-interference-and-noise ratio of the system by approximately $4 \mathrm{~dB}$ when proper power allocation among users is done, which indicates that massive MIMO is resistant against coarse quantization and that low-end ADCs can be used. 


\section{Sammanfattning}

Massiv-MIMO-basstationer (eng: Multiple-Input Multiple-Output) har visats, både i teori och praktik, besitta många av de egenskaper som framtida trådlösa kommunikationssystem kommer att behöva. De kan tillhandahålla enhetligt höga datatakter i hela täckningsområdet och simultant betjäna flera enkla mobilenheter utan att använda bredare spektrum, tätare basstationsplacering eller betydligt mer effekt än dagens basstationer. Huvudutmaningen med massiv MIMO är basstationens enorma hårdvarukomplexitet och -kostnad - varje element i den stora gruppantennen skall kunna kontrolleras individuellt och kräver sålunda sin egen radiokedja. För att massiv MIMO skall bli kommersiellt attraktiv, måste basstationen byggas av billig, enkel hårdvara. I denna avhandling undersöks hur enkla effektförstärkare och analog-till-digital-omvandlare (AD-omvandlare) påverkar massiv-MIMO-systemets prestanda. I studien av signaldistorsionen från enkla förstärkare visas det att inband-distorsionen är försumbar i massiv MIMO och att utombandsstrålningen är den begränsande faktorn som bestämmer vid vilken verkningsgrad förstärkarna kan arbeta. En förkodare som åstadkommer nerlänks-sändsignaler som har konstant envelopp i kontinuerlig tid presenteras för att möjliggöra användandet av enkla förstärkare med hög verkningsgrad. Vidare konstateras det att utombandsstrålningen är isotrop när kanalen är frekvensselektiv och när flera användare betjänas; och att den kan lobformas när kanalen är frekvensflat och när få användare betjänas. Eftersom en massiv-MIMO-basstation utstrålar mindre effekt än dagens basstationer, betyder isotrop utombandsstrålning att enkel hårdvara med sämre linearitet än vad som krävs idag kan användas i massiv MIMO. Det visas även att användandet av enbits-AD-omvandlare - de enklaste och mest strömsnåla AD-omvandlarna - i basstationen endast minskar signaltill-interferens-och-brus-förhållandet med $4 \mathrm{~dB}$ när tillbörlig effektallokering mellan användarna utförs, vilket indikerar att massiv MIMO är motståndskraftig mot grov kvantisering och att enkla AD-omvandlare kan användas. 
換約使其站上率低能出本其複部一通大 器 4 用線輻服選端影性的自敉署時訊規 可分單性射務擇功響能硬身度基間系模 於貝比要較少性放幾的件的及站頻統多 此。特求少數衰, 乎影來射基, 率所輸 類以模不功用落本可響建頻站亦資需入 系此擬比率戶信文以。造鏈成無源的多 統可數現, 時道提忽對。路本需上特輸 中見字有帶, 上出略低本。|提服質出 使, 轉基外帶服能, 端論爲高務。基 用大換站泄外務產而功文使大基多比站 。規器的露泄多生帶放探大規站個如, 模|高各露個具外信討規模的簡 : 無 多。向可用有泄號簡模天功單在論 入最另同呈戶恆露失單多線耗的其從 多簡外性現時定是真的入陣。終整理 出單表意波, 包限的功多列實端個論 系低明味束帶絡制研率出中現設覆上 統耗, 著賦外連功究放基的大備蓋或 對的如者形泄續放表大站每規, 區實 非轉果規。露時效明器有一模而域際 精換進模由呈間率, 與商個多無均上 確器行多於現信的帶模業天入需一, 量|合入大各號決內擬吸線多佔地皆 比理多規向的定失數引元出用提已 較僅的出模同預因真字力必系更供經 穩使多基多性編素對轉, 須统多高證 定系用站入; 碼。大換基單的頻數明 , 統戶些多而。爲規器站獨主譜據具 低的功使出在本使模對必可要資傳有 端信率基平文用多大須控挑源輸許 模午分低站坦指高入規以, 戰或速多 擬噪配端比衰出功多模簡因在更率未 數比, 㖇現落, 率出多單此於密、來 字降基硬用信在效的入低需硬集在無 轉低站, 基道頻率性多成要件地同線 


\section{Populärvetenskaplig sammanfattning}

Massiv Mıмo (eng: Multiple-Input Multiple-Output) är en trådlös transmissionsteknik för mobil kommunikation där basstationen använder ett stort antal samarbetande antenner för att: 1. förbättra den mottagna signalkvaliteten och på så sätt leverera en hög datatakt samt 2. kunna skicka och ta emot ett stort antal parallella dataströmmar från flera mobilenheter samtidigt över samma frekvensband och på så sätt utnyttja vårt begränsade frekvensspektrum mer effektivt. Enär massiv MIMO ställer mycket låga krav på hårdvaran i mobilenheterna, är basstationens hårdvara med de många samarbetande antennerna mycket komplex. För att denna teknik skall kunna användas i förbättringsarbetet av vårt allt viktigare samhällstäckande mobila nätverk, så att det kan stödja framtidens många nya allmännyttiga tekniska lösningar, utan att öka kostnaderna för användarna, måste basstationens hårdvara förenklas. Denna avhandling undersöker möjligheten att bygga basstationer till massiv MIMO av enkel och billig hårdvara. Genom teoretisk och numerisk analys av signalöverföringen visas att prestationen hos massiv MIMO är mycket robust mot hårdvaruimperfektioner och att många hårdvarukomponenter i basstationen inte bara kan göras enkla, utan rentav triviala. Detta innebär att det är möjligt att bygga praktiska basstationer för massiv MIMO och att tekniken kan göras kommersiellt hållbar. Allt fler och allt enklare apparater kommer att kunna betjänas trådlöst med höghastighetsuppkopplingar till rimliga priser, vilket är nödvändigt för att driva vidare den pågående utvecklingen av nya samhällsförbättrande trådlösa applikationer. 


\section{Acknowledgments}

Expedient guidance from a knowledgeable supervisor is key to a student's progress. I am very grateful to Dr. Erik G. Larsson for being this supervisor to me. The research findings herein are the fruits of his teaching. Facts and hard knowledge apart, I have also learnt from his principled attitude to research and structured way of work. Another inspiration, especially during the visit to his group in the summer 2015, has been my co-supervisor Dr. Thomas Eriksson, who is a wellspring of ideas. For education on complicated hardware, Dr. Ulf Gustavsson has been a valuable teacher. I thank Dr. Robert Heath, Jr., for expanding my research perspectives, for making research fun and for having me as his visiting student the academic year 2015-16, and Dr. Choi Junil for his careful supervision during this visit. The research visit to Dr. Heath's group at the University of Texas at Austin was made possible by the generous scholarships from the Fulbright Commission, Ericsson's Research Foundation, Stiftelsen Blanceflor and Ingenjörsvetenskapsakademien's Hans Werthén Fond.

The steady support from my family is what makes it possible to always persist in my endeavors. During my studies, I have come into contact with many colleagues-motivating, interesting, smart. It is in their presence I have been able to produce this piece of work. I recognize the privilege that, in our country, where everybody is encouraged to pursue learning and where anybody truly is allowed access to higher education, the path to knowledge and research, albeit strenuous, is straight. I therefore bow to my family, every member of the research groups Kommunikationssystem in Linköping and WSIL in Austin and the people of Sweden-it is to you I owe this thesis.

Christopher Mollén

Linköping, August 2016 
xii 


\section{Contents}

1 Introduction 1

1.1 Massive MIMO . . . . . . . . . . . . . . 2

1.2 Contributions of the Thesis ............ 4

2 Communication Theory 9

2.1 Signal Representations . . . . . . . . . . . . . . 9

2.2 The Wireless Communication Channel . . . . . . . . . 14

2.3 Multi-User MIMO . . . . . . . . . . . . . . . . . . 19

2.4 Channel Estimation . . . . . . . . . . . . . . 21

2.5 Precoding and Combining . . . . . . . . . . 26

3 Hardware 35

3.1 Transmitter Design $\ldots \ldots \ldots \ldots \ldots \ldots$

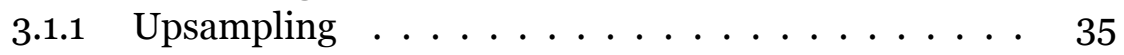

3.1 .2 Distortion Compensation . . . . . . . . . . 36

3.1.3 Upconversion . . . . . . . . . . . . . . . . 38

3.1 .4 Amplification . . . . . . . . . . . . . . . . 38

3.2 Receiver Design . . . . . . . . . . . . 42

4 Future Work $\quad 47$

$\begin{array}{ll}\text { Bibliography } & 49\end{array}$

Included Papers $\quad 55$

A Waveforms for the Massive MIMO Downlink 57

1 Introduction . . . . . . . . . . . . . . 59

2 System Model . . . . . . . . . . . . . . . 62

3 Downlink Transmission . . . . . . . . . . . . . . . 67

3.1 Achievable Data Rates . . . . . . . . . . 68 
3.2 Linear Precoding Techniques . . . . . . . . . . . 71

3.3 Low-PAR Precoding Techniques . . . . . . . . . . 75

3.4 Power Allocation among Users . . . . . . . . . . . 77

3.5 Single-Carrier vs. OFDM Transmission . . . . . . . . 79

4 Numerical Evaluations of Rate . . . . . . . . . . . . . 80

4.1 Effects of Nonlinear Power Amplifiers . . . . . . . . 81

4.2 Data Rate and Power Consumption . . . . . . . . 85

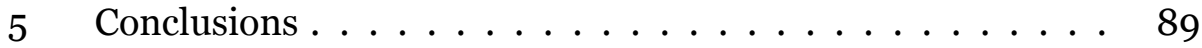

Appendix: Proof of Proposition 1 . . . . . . . . . . . 90

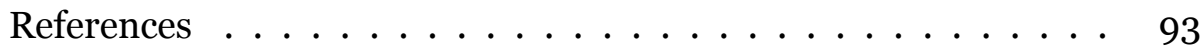

B Continuous-Time Constant-Envelope Precoding 97

1 Introduction . . . . . . . . . . . . . . . . 99

2 System Model . . . . . . . . . . . . . . . . . . 100

3 The Constant-Envelope MIMO Channel . . . . . . . . . . . 101

4 The CTCE Massive MIMO Downlink . . . . . . . . . . . . . . 103

$4.1 \quad$ CTCE Precoding . . . . . . . . . . . . . . 103

4.2 Constant-Envelope Modulation . . . . . . . . . 107

5 Achievable Rate . . . . . . . . . . . . . . . . . . . . 107

6 Numerical Analysis of the CTCE Precoder . . . . . . . . . . . 108

7 Conclusion . . . . . . . . . . . . . . . . 112

Acknowledgment . . . . . . . . . . . . . . . . . . 112

References ...................... 112

C Out-of-Band Radiation from MIMO Arrays 115

1 Introduction . . . . . . . . . . . . . . 117

2 Notation . . . . . . . . . . . . . . . . . 119

3 Downlink System Model . . . . . . . . . . . . . . . . . . . . . 119

4 Base Station Radiation Pattern . . . . . . . . . . . . . . . 120

5 Measures of Out-of-Band Radiation . . . . . . . . . . . . 123

5.1 The Traditional Single-Antenna Setting . . . . . . . . 123

5.2 The Multi-Antenna Setting . . . . . . . . . . . . . 124

5.3 Worst-Case Out-of-Band Radiation . . . . . . . . . 126

6 Numerical Examples . . . . . . . . . . . . . . . . . . . . . 127

6.1 Assumptions of the Numerical Analysis . . . . . . . . 127

6.2 Numerical Results . . . . . . . . . . . . . . . . . 128

7 Conclusions . . . . . . . . . . . . . . . 130

Acknowledgment . . . . . . . . . . . . . . . . . . . 132

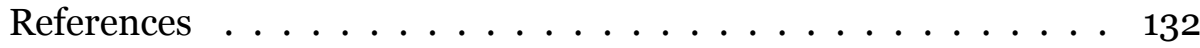


D Massive MIMO with One-Bit ADCs 135

1 Introduction . . . . . . . . . . . . . . . 137

2 System Model . . . . . . . . . . . . . . . . . . 140

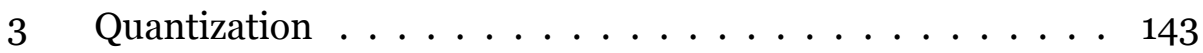

4 Channel Estimation . . . . . . . . . . . . . . . . 146

5 Uplink Data Transmission . . . . . . . . . . . . 151

$5.1 \quad$ Receive Combining . . . . . . . . . . . . 151

5.2 Quantization Error and its Effect on Single-Carrier and OFDM Transmission . . . . . . . . . . . . 152

5.3 Achievable Rate . . . . . . . . . . . . . 155

6 Numerical Examples . . . . . . . . . . . . . . . . . . . 161

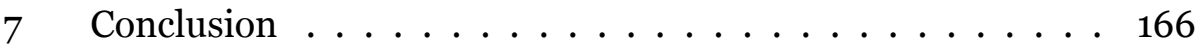

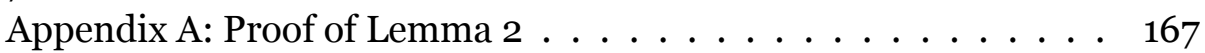

Appendix B: Proof of Lemma $3 \ldots \ldots$

Appendix C: Proof of Theorem $1 \ldots \ldots$. . . . . . . . . . . . . 168

References . . . . . . . . . . . . . . . . . . 169 



\section{Chapter 1}

\section{Introduction}

A teenager heads to a distant part of the Globe to live and study in a foreign culture that is largely different from the one she grew up in. Such a venture is no longer seen as a great expedition, but rather as a commonplace trip. Why is that? - The parents can talk to and see their child over their smartphone at anytime using a messenger application. The teenager has beforehand got a good grasp of her future living circumstances from the Internet, and she can navigate and obtain information about her new neighborhood in her own language on the go using GPS and the cellular network. The possibility to communicate electronically has obviously changed modern life and become an integral part of it. With this small example, I want to point out one of its greatest benefits: uncensored global connectivity brings people closer, promotes intercultural understanding and enables borderless exchange of ideas, which in turn has the potential to reduce the risk of conflicts-both global and personal-and speed up scientific and cultural development. With this as motivation, the research in this thesis aims at giving a better understanding of some practical issues of massive MIMO-the technology that has the potential to replace today's base stations and enhance our wireless communication systems to cope with the increasing data traffic load, higher user density and new functionality requirements that the surging use of wireless technology is expected to generate in a near future. A better understanding of how to implement the theoretical idea of massive MIMO in practical hardware is essential for its adoption in the continued development of our networked society. 


\subsection{Massive MIMO}

Massive MIMO, as was envisioned in [1], is a communication technology, where a base station is equipped with hundreds of antennas. The many antennas are used to concurrently serve multiple single-antenna users over the same time and frequency resource. It can increase the data rate that the users are served with by orders of magnitude compared to conventional systems without using more frequency spectrum, and possibly also without using more power. Furthermore, massive MIMO can provide uniformly good service to all users in a large area, both users far away and users near the base station. These qualities make massive MIMO a good choice of technology for the evolution of today's wireless communication systems to meet the new and greater demands of the future $[2,3]$.

The main qualities of massive MIMO are:

array gain that grows with the number of antennas, which improves the signal quality and lowers the amount of power that has to be radiated.

spatial multiplexing that makes it possible to concurrently serve multiple users at the same time over the same frequencies, which enables high sum rates.

simple handsets that only have a single antenna and do not perform any complicated channel equalization, which can allow for the integration of small low-power, low-cost devices into the cellular system.

linear signal processing that makes the base station baseband processing simpler and feasible in terms of computational complexity, which, in many cases, gives a performance that is close to the optimal highly complex dirty paper coding [4].

Prototypes of massive MIMO base stations have already been built. Noteworthy among academic research institutes are the testbeds developed at: Rice University (Argos) [5], Lund University [6] and Bristol University [7], and among industrial research institutes are the ones at: Samsung, Nutaq and Facebook. These testbeds have shown that the theoretical benefits of massive MIMO are real. However, their implementation has been expensive. To bring cost down and make massive MIMO commercially viable, its base stations have to be built from inexpensive, low-end hardware. The effects on the 
system of using low-end power amplifiers and analog-to-digital converters (ADCs) are studied in this thesis. It is found that massive MIMO is very robust against the imperfections of low-end hardware. It is further shown that the error caused by both amplifiers and ADCs typically combines non-coherently, which means that the performance degradation becomes small when the number of antennas is large, also when linear signal processing is used. Since the number of antennas is large, this thesis supports the claim that low-end hardware can be used in massive MIMO.

Previous work has dealt with hardware imperfections by modeling it as a signal distortion that collectively can be described by a simple parametric function. This approach is used in [8] and [9] for example. In [8], both the downlink and uplink are studied and the impact of the hardware is treated as additive noise. In [9], the uplink is studied and a refined model treats the hardware imperfections of the base station as multiplicative phase drifts, additive distortion noise and noise amplification, which should model the effects of the ADCs, LNAS and the oscillators. A similar approach to [8] is used to model hardware imperfections in [10], where the results are verified against simulations with more sophisticated measurement-based models. These studies showed that the hardware quality can be degraded in massive MIMO and that the more antennas the base station has the less accurate the hardware can be allowed to be. What has not been analyzed in previous work and that this thesis studies, is how signals with different amplitude distributions compare when the effects of low-end hardware are taken into account, the effect of imperfect hardware on out-of-band radiation, how out-of-band radiation behaves spatially and the feasibility of using low-end ADCs with extremely low resolution.

A unique feature of massive MIMo is the possibility to use spatial degrees-offreedom to do crest-factor reduction without causing any distortion in the receive signal. This crest-factor reduction comes at the cost of an increased transmit power however. When crest-factor reduction is done together with the symbol precoding, so called low-PAR (peak-to-average ratio) precoding, the amplitude distribution of the transmit signals can be made more hardware friendly. The first low-PAR precoding method for massive MIMO was presented in [11], where a single-user precoder was proposed. This method was extended, first to a multi-user precoder in [12] and then to a multi-user precoder for frequency-selective channels in [13], which is called the discrete-time constant-envelope precoder in this thesis. Another type of low-PAR precoder is presented in [14], which can control the trade-off 
between crest-factor reduction and increased transmit power. Low-PAR precoders are especially interesting for massive MIMO, because conventional precoders result in transmit signals with high PAR that are heavily affected by nonlinear low-end hardware. In this thesis, low-PAR precoding is evaluated against conventional precoding methods in terms of spectral efficiency and the power consumption of the amplifiers of the base station. A new low-PAR precoder, the continuous-time constant-envelope precoder, is also proposed, which produces continuous-time transmit signals with o dB PAR. Such signals can be amplified with high power efficiency in highly nonlinear inexpensive amplifiers without causing distortion and spectral regrowth.

An ostensible weakness of an additive noise model for hardware impairments is that many types of signal distortion are deterministic-given the input signal, the output can be determined. Even if the input signal is modeled as stochastic, the distortion will depend on the input signal and its distribution will not be accurately described as an independent Gaussian. This is especially true for low-end ADCs with low resolutions and the extreme one-bit ADCs. To verify that the scaling laws that were derived in, e.g., [9] also can be applied to one-bit ADCs, the quantization of the ADC has to be studied in detail. The use of one-bit ADCs in massive MIMO, was initially studied in [15]. The use of onebit ADCs had previously also been studied for MIMO systems in, e.g., [16, 17] and for millimeter wave MIMO systems in, e.g., [18]. The feasibility of one-bit ADCs, in terms of achievable rate, was studied in [17], where it was shown that one-bit ADCs only lead to a small capacity reduction in a MIMO system at high noise levels, and in [19], where it was shown that the capacity at low noise levels is very high and scales linearly with the number of receive antennas. A detector with near maximum-likelihood performance for one-bit ADC systems was proposed in [20]. However, the computational complexity of the detector becomes prohibitive when the channel is frequency selective. Therefore, this thesis investigates the use of low-complexity linear receivers for massive MIMO systems with one-bit ADCs. This thesis derives achievable rates for such systems and shows that, if the receive filter can be implemented as an analog filter, the effective SINR loss that one-bit ADCs cause the transmission typically is approximately $4 \mathrm{~dB}$ compared to unquantized systems.

\subsection{Contributions of the Thesis}

This thesis explains and evaluates some of the practical issues of massive MIMO that are not captured by the conventional idealistic linear system model. 
It is found that massive MIMO is quite robust to nonlinear imperfections and that issues, such as amplifier distortion and coarse quantization, naturally vanishes as the number of base station antennas is increased. Below follows a more detailed presentation of the particular contributions of each paper that is included in this thesis.

All papers are written by the first author himself based on ideas that have sprung from discussions with the co-authors of each paper. The theoretical and empirical results in the papers are derived and implemented by the first author himself. The first author recognizes the great contribution of all the co-authors, who have spent a significant amount of time on supervising his work, revising his texts and have shared their deep professional expertise with him.

\section{Paper A: Waveforms for the Massive MIMO Downlink: Amplifier Efficiency, Distortion and Performance}

Authored by: Christopher Mollén, Erik G. Larsson and Thomas Eriksson

Published in: IEEE Transactions on Communications, April 2016.

The massive MIMO downlink relies on precoded transmission to spatially multiplex individual data streams to different users. Data can be transmitted either over the whole spectrum with single-carrier transmission or over separate subcarriers with OFDM transmission. Furthermore, precoding can be done in many different ways: by conventional methods that do not consider the effect of the nonlinear power amplifier and by hardware-aware precoders that produce hardware-friendly signals, which allow the amplifier to be operated with higher power efficiency. In this paper, different transmission and precoding methods are evaluated in terms of spectral efficiency and power consumption of the amplifiers. It is found, that conventional and hardware-friendly precoders result in approximately the same amplifier power consumption when operated at the same spectral efficiency. It is also observed that single-carrier and OFDM transmission have the same performance in massive MIMO in terms of achievable data rates and that both transmission methods result in signals with similar PAR, which is not the case in conventional communication systems, where usually only OFDM suffers from high PAR. The two transmission techniques therefore also result in the same amplifier power consumption. 


\section{Paper B: Multiuser MIMO Precoding with Per-Antenna Continuous-Time Constant-Envelope Constraints}

Authored by: Christopher Mollén and Erik G. Larsson

Published in: The Proceedings of the International Workshop on Signal Processing Advances in Wireless Communications, pp. 261 - 265, June 2015.

From a signal generation point of view, continuous-time signals with constant envelope are the most preferable. Such signals allow for highly power efficient and inexpensive radio chain designs. For example, class $\mathrm{C}$ or switched mode amplifiers could be used without causing prohibitive signal distortion. This paper presents a precoder for the massive MIMO downlink that results in transmit signals with continuous-time constant envelopes. It is shown that there is a trade-off between excess bandwidth and performance. In one example system, at low data rates and an excess bandwidth of $40 \%$, the proposed precoder needs $3 \mathrm{~dB}$ more radiated power to achieve the same data rate as conventional zero-forcing precoding. It is argued that this extra radiated power might be compensated for by the increased power efficiency and the decreased complexity of the hardware.

\section{Paper C: Out-of-Band Radiation Measure for MIMO Arrays with Beamformed Transmission}

Authored by: Christopher Mollén, Ulf Gustavsson, Thomas Eriksson and Erik G. Larsson

Published in: The Proceedings of the IEEE International Conference on Communications, May 2016.

Nonlinear hardware in the transmitter causes the base station to radiate power outside the allocated band, so called out-of-band radiation. When the signal is transmitted over a shared wireless medium, the out-of-band radiation can disturb other victim systems operating in adjacent frequency bands. In a MIMO system, there is also the risk that the radiation is beamformed and builds up coherently at the victim, which would amplify the disturbance. In this paper, we study the spatial behavior of the out-of-band radiation. It is found that the out-of-band radiation is beamformed to some extent and that this beamforming becomes more prominent the less frequency selective the channel is and the fewer users that are served by the system. In a frequency-selective multiuser channel however, the out-of-band radiation is close to isotropic. Further, it is observed that the array gain of the outof-band radiation is smaller than the array gain of the desired signal. Since the array gain of the desired signal allows for reduced radiated power, the 
total effective out-of-band radiation from a MIMO array is lower than from a conventional single-antenna transmitter when they are operated at the same spectral efficiencies and with the same linearity requirements. Large MIMO arrays thus allow for less linear hardware, which increases power efficiency and reduces the cost of the array.

\section{Paper D: Uplink Performance of Wideband Massive MIMO with One-Bit ADCs}

Authored by: Christopher Mollén, Junil Choi, Erik G. Larsson and Robert W. Heath, Jr.

Submitted to: IEEE Transactions on Wireless Communications

The base station in a massive MIMO system requires a large number of radio chains-each antenna needs one. This makes the hardware cost and the power consumption large. One way to reduce hardware complexity and power consumption of the receiver is to use ADCs with low resolutions. This paper investigates the feasibility, in terms of achievable rate, of letting the base station use ADCs with the lowest possible resolution-one-bit ADCs operated at the baudrate. Such ADCs are very easy to implement, consume negligible amounts of power and do not require any advanced automatic gain control. It is shown that, also with one-bit ADCs, channel estimation and symbol detection can be done with linear signal processing, as long as the receive filter can be implemented as an analog filter. Furthermore, it is shown that the use of one-bit ADCs leads to an SINR loss of approximately $4 \mathrm{~dB}$ at low spectral efficiencies, which could be overcome by using a factor 2.5 more base station antennas. 
1 Introduction 


\section{Chapter 2}

\section{Communication Theory}

This chapter introduces the fundamentals of the communication theory that is used in this thesis. The well-versed reader may skip through this chapter.

\subsection{Signal Representations}

The exact signal that is transmitted in a communication system can take many different forms. In the study of a general communication system however, the exact nature of a specific signal is seldom interesting. Instead, only properties of the signal, such as its power, its spectrum and the statistics of its amplitude, are relevant. For this reason, the signals in a communication system are usually modeled as stochastic processes [21]. The power of such a process $x(t)$ is defined by

$$
P(x(t)) \triangleq \lim _{t_{0} \rightarrow \infty} \frac{1}{2 t_{0}} \mathrm{E}\left[\int_{-t_{0}}^{t_{0}}|x(t)|^{2} \mathrm{~d} t\right] .
$$

The spectral properties of the signal are captured by its power spectral density if it exists, which is a function $S(f)$ such that

$$
\int_{-\infty}^{\infty} S(f)|u(f)|^{2} \mathrm{~d} f=P\left(\int_{-\infty}^{\infty} u(\tau) x(t-\tau) \mathrm{d} \tau\right),
$$

for all integrable functions $u(\tau): \mathbb{R} \rightarrow \mathbb{C}$ with Fourier transform $u(f)$. 
If, as a special case, $u(\tau)$ is taken to be a narrow bandpass filter around $f_{0}$, it can be seen that the power spectral density $S\left(f_{0}\right)$ can be interpreted as the power of its stochastic process at this frequency, normalized by the bandwidth of the filter. If $x(t)$ is a wide-sense stationary process, i.e. its autocorrelation

$$
R(\tau) \triangleq \mathrm{E}\left[x^{*}(t) x(t+\tau)\right]
$$

and mean $\mathrm{E}[x(t)]$ do not depend on $t$, then the power spectral density is given as the function $S(f)$ whose inverse Fourier transform equals the autocorrelation:

$$
R(\tau)=\int_{-\infty}^{\infty} S(f) e^{j 2 \pi f \tau} \mathrm{d} f, \quad \forall \tau \in \mathbb{R} .
$$

It can be shown that, if it exists, the power spectral density is unique and a realvalued non-negative function (except, possibly on a set of Lebesgue measure zero). In the included papers, signals are often treated as ordinary nonstochastic functions to simplify the exposition and no notational difference is made between signals that are stochastic and signals that are deterministiccontext has to distinguish the two.

A wireless communication medium, such as air or empty space, is commonly shared between many systems. To avoid interference, each system is usually allocated a frequency band $\left[ \pm f_{\mathrm{c}}-B / 2, \pm f_{\mathrm{c}}+B / 2\right.$ ] of its own of some width $B$ around a carrier frequency $f_{\mathrm{c}}$. The typical transmit signal used for wireless communication is thus a real-valued signal $x_{\mathrm{pb}}(t)$ whose energy is zero outside this band, i.e. a signal whose power spectral density $S_{\mathrm{pb}}(f)=0$ when $|f| \notin\left[f_{\mathrm{c}}-B / 2, f_{\mathrm{c}}+B / 2\right]$. Such a signal is called a passband signal.

The wireless communication medium can be modeled as a linear system whose properties change slowly in relation to the time duration of the transmit signal. Therefore it is assumed that the signal $y_{\mathrm{pb}}(t)$ that is received during the transmission is given by the transmit signal and the impulse response $g_{\mathrm{pb}}(\tau)$ of the channel in the following way

$$
y_{\mathrm{pb}}(t)=\sqrt{P} \int_{-\infty}^{\infty} g_{\mathrm{pb}}(\tau) x_{\mathrm{pb}}(t-\tau) \mathrm{d} \tau+z_{\mathrm{pb}}(t),
$$

where we let $P$ denote the transmit power by requiring that

$$
P\left(x_{\mathrm{pb}}(t)\right)=1,
$$

and where $z_{\mathrm{pb}}(t)$ is a noise term that models the thermal noise of the receiving hardware. The noise is modeled as a Gaussian stochastic process that is 


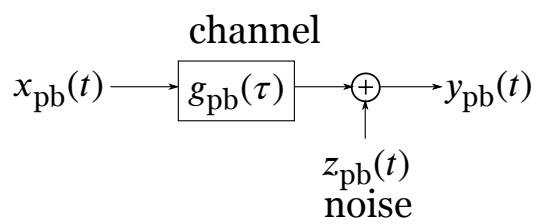

Figure 1: General communication system

white over the allocated band, i.e. its power spectral density is constant for $|f| \in\left[f_{\mathrm{c}}-B / 2, f_{\mathrm{c}}+B / 2\right]$. The constant spectral height is denoted $N_{0}$. The general setting is depicted in Figure 1.

The channel introduces two distortion effects: large-scale fading and smallscale fading. The large-scale fading is the signal attenuation due to both the distance the signal has traversed and the materials that the signal has penetrated on its way to the receiver. The small-scale fading is the aggregate amplitude and phase distortion that stems from multi-path propagation, where the received signal is the superposition of many copies of the same signal with different time delays. By denoting the attenuation due to largescale fading by $\beta \in[0,1]$ and the effects of small scale fading by $h_{\mathrm{pb}}(\tau)$, the impulse response of the channel can be factorized as follows:

$$
g_{\mathrm{pb}}(\tau)=\sqrt{\beta} h_{\mathrm{pb}}(\tau) .
$$

The large-scale fading changes very little over the course of the transmission. It is therefore relatively easy to estimate and is assumed to be known to both transmitter and receiver. The small-scale fading, on the other hand, changes slowly over the course of the transmission and has to be estimated. The factorization in (7) is thus helpful to distinguish what is known and what has to be kept track of in our models.

The rough range, in which the carrier frequency lies, determines certain propagation characteristics of the wireless medium, e.g., the amount of path loss, penetration loss and molecular absorption that can be expected [22]. Other than that, the carrier frequency is of little importance for the theoretical study of the general communication system in Figure 1. It is therefore common practice to represent the physical real-valued passband signal by its complex baseband equivalent:

$$
x(t)=\mathrm{LP}_{B / 2}\left(x_{\mathrm{pb}}(t) e^{-j 2 \pi f_{\mathrm{c}} t}\right),
$$

where $\mathrm{LP}_{B / 2}$ denotes an ideal lowpass filter with cutoff frequency $B / 2$, see [23] for a thorough introduction to the baseband model. In the baseband notation, 
the passband signal $x_{\mathrm{pb}}(t)$ at any time instant $t$ is represented by a complex number that naturally represents its phase and envelope by $\arg (x(t))$ and $|x(t)|$ respectively. Note that the transform in (8) is invertible:

$$
x_{\mathrm{pb}}(t)=2 \mathfrak{R e}(x(t)) \cos \left(2 \pi f_{\mathrm{c}} t\right)-2 \mathfrak{I m}(x(t)) \sin \left(2 \pi f_{\mathrm{c}} t\right)
$$

and that the power spectral density of the baseband signal $S(f)=S_{\mathrm{pb}}\left(f+f_{\mathrm{c}}\right)$ in the band $f \in[-B / 2, B / 2]$ and $S(f)=0$ outside that band. Furthermore, the received signal in $(5)$ is given by

$$
y(t)=\sqrt{\beta P} \int_{-\infty}^{\infty} h(\tau) x(t-\tau) \mathrm{d} \tau+z(t)
$$

in the equivalent base band representation, where the baseband signals $y(t)$, $z(t)$ and $h(\tau)$ are defined in the same way as $x(t)$ in (8). Note that the thermal noise $z(t)$ then becomes a realization of a complex circularly symmetric Gaussian stochastic process, whose power spectral density is equal to the constant $N_{0}$ in the band $f \in[-B / 2, B / 2]$.

A common way of encoding information onto the transmit signal is to map it onto a sequence of complex values $\{x[n]\}$ first. These values are then pulseamplitude modulated by a transmit filter $p^{\prime}(\tau)$ into the baseband transmit signal:

$$
x(t)=\sum_{n} p^{\prime}(t-n T) x[n],
$$

where $T$ is called the symbol duration. To make the transmit signal fit within its allocated band, the filter $p(\tau)$ has to be bandlimited to within $[-B / 2, B / 2]$. Further, if $\{x[n]\}$ is a series of i.i.d. random variables such that $\mathrm{E}\left[|x[n]|^{2}\right]=1$, then the energy of the filter has to be

$$
\int_{-\infty}^{\infty}\left|p^{\prime}(\tau)\right|^{2} \mathrm{~d} \tau=T
$$

to make the transmit signal fulfill its power constraint (6). To again obtain a sequence of complex values, the reverse operation, demodulation, is performed on the receive signal

$$
y^{(\kappa)}[n]=\int_{-\infty}^{\infty} p(\tau) y(n T / \kappa-\tau) \mathrm{d} \tau,
$$

where $p(\tau)$ is the receive filter and $\kappa$ the oversampling factor. When $\kappa=1$, the signal is given in symbol-sampled time and the superscript is omitted: 


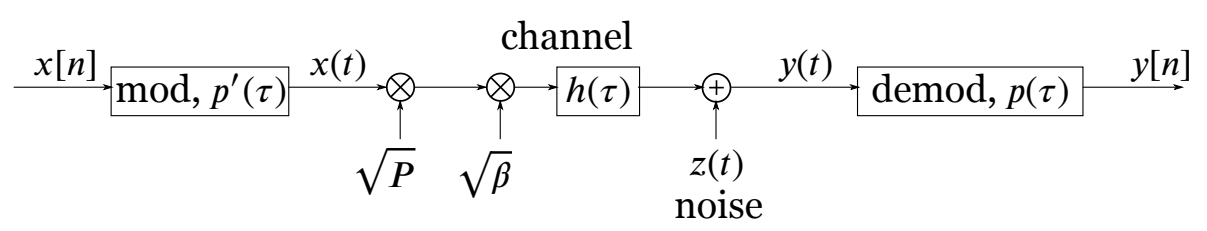

(a) continuous time

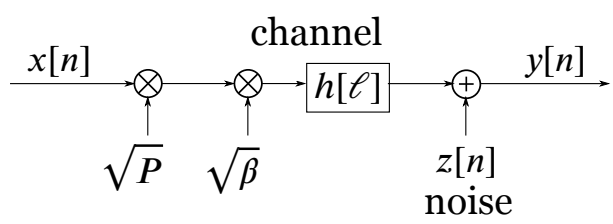

(b) symbol-sampled discrete time

Figure 2: Equivalent baseband model of a general communication system

$y[n] \triangleq y^{(1)}[n]$. When pulse-amplitude modulation and demodulation is used, the communication channel given in (10) and shown in Figure 2(a) can equivalently be given in symbol-sampled time as in Figure 2(b), where the received signal is given by:

$$
y[n]=\sqrt{\beta P} \sum_{\ell=-\infty}^{\infty} h[\ell] x[n-\ell]+z[n],
$$

the discrete-time channel impulse response is given by

$$
h[\ell]=\int_{-\infty}^{\infty} \int_{-\infty}^{\infty} p^{\prime}(\tau) h\left(\tau^{\prime}-\tau\right) p\left(\ell T-\tau^{\prime}\right) \mathrm{d} \tau \mathrm{d} \tau^{\prime}
$$

and the discrete-time noise is

$$
z[n]=\int_{-\infty}^{\infty} p(\tau) z(n T-\tau) \mathrm{d} \tau .
$$

Since the communication model in (14) is equivalent to (1) when modulation and demodulation are done according to (11) and (13), communication systems are usually studied in symbol-sampled time for simplicity. However, when nonlinear systems are studied, such as in Papers A, B and C, oversampled signals have to be considered because nonlinearities can cause spectral regrowth that results in undesired aliasing in the sampling.

A Nyquist pulse of parameter $T$ is a pulse whose Fourier transform $\Gamma(f)$ fulfills

$$
\sum_{n=-\infty}^{\infty} \Gamma\left(f-\frac{n}{T}\right)=\text { constant }
$$


for all $f$, except possibly for $f$ in some set of Lebesgue measure zero. To make the discrete-time noise $z[n]$ white, the receive filter is usually chosen as a root-Nyquist pulse of parameter $T$, i.e. a pulse whose self-convolution $\int p(\tau) p^{*}(\tau-t) \mathrm{d} \tau$ is a Nyquist pulse of parameter $T$. When the receive filter is a root-Nyquist pulse and has the energy

$$
\int_{-\infty}^{\infty}|p(\tau)|^{2} \mathrm{~d} \tau=\frac{1}{T}
$$

then $z[n] \sim \mathscr{C} \mathcal{N}\left(0, N_{0} / T\right)$ i.i.d. To maximize the signal-to-noise ratio (SNR) ${ }^{1}$, i.e. the power of the desired signal in relation to the power of the noise

$$
\frac{\mathrm{E}\left[|y[n]-z[n]|^{2}\right]}{\mathrm{E}\left[|z[n]|^{2}\right]},
$$

the transmit filter is matched to the receive filter [24], i.e. $p^{\prime}(\tau)=T p^{*}(-\tau)$, where the transmit filter is scaled by $T$ so that the power constraint (12) holds. A common choice of filters in practical communication systems are the root-raised cosine filters, which have a good trade-off between narrow bandwidth and short delays. The root-raised cosine filters are a family of filters parameterized by their excess bandwidth $\alpha \triangleq T B-1$, which is a measure of how much wider the bandwidth $B$ is compared to the baudrate $1 / T$. Note that the bandwidth of a Nyquist pulse never can be smaller than the baudrate, but that the bandwidth of the filter usually is chosen as close to the baudrate as possible to use the spectrum efficiently-a small excess bandwidth is desired. For the root-raised cosines, $\alpha$ can vary between 0 and 1 . The common choice $\alpha=0.22$, which means that the bandwidth of the pulse is $22 \%$ wider than the baudrate, is used to evaluate the theoretical results in Papers A, B and C.

\subsection{The Wireless Communication Channel}

When a signal is transmitted, it will bounce around from reflective materials in the surrounding environment and the transmitted signal will travel many

\footnotetext{
${ }^{1}$ It is desirable to leave the channel equalization to the digital part of the radio chain. For this reason, "maximize the SNR" is taken to mean to maximize the average SNR over all channel realizations at this point, i.e. to ensure the highest possible SNR if the channel were flat.
} 


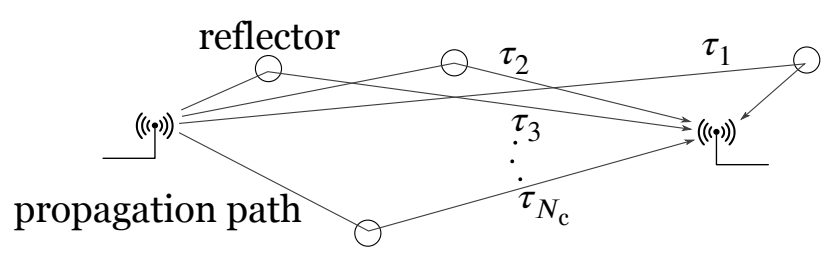

Figure 3: Multipath propagation

different paths to the receiver. Therefore, the received signal will be a superposition of many differently attenuated and delayed copies of the transmitted signal. This is illustrated in Figure 3.

If the number of paths is $N_{\mathrm{c}}$, then the receive signal can be written:

$$
y_{\mathrm{pb}}(t)=\sqrt{\beta} \sum_{i=1}^{N_{\mathrm{c}}} \alpha_{i} x_{\mathrm{pb}}\left(t-\tau_{i}\right),
$$

where $\beta$ is the large-scale fading and $\alpha_{i}$ the real-valued amplitude scaling and $\tau_{i}$ the delay of the $i$-th path [23]. Using the baseband notation, the relation in (20) becomes

$$
y(t)=\sqrt{\beta} \sum_{i=1}^{N_{\mathrm{c}}} \alpha_{i} e^{-j 2 \pi f_{\mathrm{c}} \tau_{i}} x\left(t-\tau_{i}\right) .
$$

Hence, the channel impulse response is

$$
h(\tau)=\sum_{i=1}^{N_{\mathrm{c}}} \alpha_{i} e^{-j 2 \pi f_{\mathrm{c}} \tau_{i}} \delta\left(\tau-\tau_{i}\right)
$$

and, according to (15), the discrete-time channel becomes

$$
h[\ell]=\sum_{i=1}^{N_{\mathrm{c}}} \gamma\left(\ell T-\tau_{i}\right) \alpha_{i} e^{-j 2 \pi f_{\mathrm{c}} \tau_{i}},
$$

where $\gamma(\tau) \triangleq \int p^{\prime}(t) p(\tau-t) \mathrm{d} t$ is the aggregate transmit-receive filter. We can assume that $\left\{\gamma\left(n T-\tau_{i}\right)\right\}_{n=-\infty}^{\infty}$ is a sequence with only a few non-zero taps around $n_{i}=\operatorname{round}\left(\tau_{i} / T\right)$, since $\gamma(\tau)$ is a Nyquist pulse. The support of the discrete-time impulse response of the channel is thus approximated by the difference

$$
\sigma_{\tau} \triangleq \max _{i, i^{\prime}}\left(\tau_{i}-\tau_{i^{\prime}}\right)
$$


which is one measure of the delay spread of the channel. The number $L$ of non-zero taps in the discrete-time channel is then roughly

$$
L \approx \frac{\sigma_{\tau}}{T}
$$

A channel, where the discrete-time channel impulse response has $L=1$, is called a frequency-flat channel, because the discrete-time Fourier transform of $h[n]$ is a constant. If $L>1$, the channel is frequency selective, because the discrete-time Fourier transform varies with frequency. The delay of a path $i$ is a function of the length $d_{i}$ of that path: $\tau_{i}=d_{i} / c$, where $c$ is the speed of the signal (usually the speed of light). For example, in an outdoor environment, where the wireless communication system is supposed to cover an area of diameter $1000 \mathrm{~m}$, it would be reasonable to assume that the maximum difference in path lengths $\max \left(d_{i}-d_{i^{\prime}}\right) \approx 1000 \mathrm{~m}$. In such an environment, the delay spread is approximately

$$
\sigma_{\tau} \approx \frac{1}{c} \max _{i, i^{\prime}}\left(d_{i}-d_{i^{\prime}}\right) \approx 3.3 \mu \mathrm{s},
$$

where $c=300 \mathrm{Mm} / \mathrm{s}$, the speed of light, was assumed. The discrete-time channel is thus approximately frequency flat $(L=1)$ in a system with baudrate $1 / T=300 \mathrm{kHz}$, and frequency selective with $L=67$ taps with baudrate $20 \mathrm{MHz}$.

Movements in the propagation environment will change the time delays, amplitude scalings and the number of paths of the channel. We have previously claimed that the channel impulse response is roughly static during the course of the transmission. This claim is equal to claiming that all the reflectors and the transmitter and receiver are still during the course of the transmission. This is of course not the case for a general channel. If the channel changes in a way that can be accurately tracked over time however, a static channel model or a dynamic one are practically equivalent. The time, which the channel can be accurately tracked, and during which the static channel model thus can be used, is called the coherence time. It can be derived by letting the length of path $i$ at time $t$ be $d_{i}(t)$. Then the delay of that path is given by $\tau_{i}=d_{i}(t) / c$. If $v_{i}$ is the constant rate, at which the length $d_{i}(t)$ shrinks or elongates over time due to movements in the environment, then the Doppler shift of path $i$ is given by $f_{i} \triangleq v_{i} / \lambda$, where the wavelength of the signal is given by $\lambda \triangleq c / f_{\mathrm{c}}$. By defining $\alpha_{i}^{\prime}[\ell] \triangleq \gamma\left(\ell T-\tau_{i}\right) \alpha_{i}$, the $\ell$-th tap of the discrete-time channel in (23) can be written as

$$
h[\ell]=\sum_{i=0}^{N_{\mathrm{c}}} \alpha_{i}^{\prime}[\ell] e^{-j 2 \pi\left(d_{i}(0) / \lambda+f_{i} t\right)},
$$


where $d_{i}(t)=d_{i}(0)+v_{i} t$ is used to write

$$
\begin{aligned}
f_{\mathrm{c}} \tau_{i} & =\left(d_{i}(0)+v_{i} t\right) f_{\mathrm{c}} / c \\
& =\left(d_{i}(0)+v_{i} t\right) / \lambda \\
& =d_{i}(0) / \lambda+f_{i} t .
\end{aligned}
$$

To gain some intuition, first assume that only one path $i=1$ contributes to $\operatorname{tap} \ell$. Then

$$
h[\ell]=\underbrace{\alpha_{1}^{\prime}[\ell] e^{-j 2 \pi d_{1}(0) / \lambda}}_{\text {constant }} e^{-j 2 \pi f_{1} t} .
$$

Even though the channel coefficient is not constant over time, it can easily be tracked because the evolution is a phase shift with constant rate $f_{1}$, as long as the value at some time $t=t_{0}$ is known. Now assume that only two of the paths $i=1,2$ significantly contribute to tap $\ell$. The following reasoning can be extended to arbitrary many taps, but it would only obfuscate the exposition and lead to little additional insight. Channel tap $\ell$ is thus

$$
\begin{aligned}
h[\ell] & =\alpha_{1}^{\prime}[\ell] e^{-j 2 \pi\left(d_{1}(0) / \lambda+f_{1} t\right)}+\alpha_{2}^{\prime}[\ell] e^{-j 2 \pi\left(d_{2}(0) / \lambda+f_{2} t\right)} \\
& =(\underbrace{\alpha_{1}^{\prime}[\ell] e^{-j 2 \pi d_{1}(0) / \lambda}}_{\text {constant }}+\underbrace{\alpha_{2}^{\prime}[\ell] e^{-j 2 \pi d_{2}(0) / \lambda}}_{\text {constant }} \underbrace{e^{-j 2 \pi\left(f_{2}-f_{1}\right) t}}_{\text {not constant }}) e^{-j 2 \pi f_{1} t} .
\end{aligned}
$$

Now the evolution over time is no longer a constant-rate phase shift. Knowledge of the value of $h[\ell]$ at some time $t=t_{0}$ is no longer enough to track the channel coefficient over time. (With knowledge of $\alpha_{1}^{\prime}[\ell]$ and $\alpha_{2}^{\prime}[\ell]$, it would be possible but they are difficult to estimate.) The time period, during which it is possible to keep track of the channel coefficient is therefore approximated by how long also the term

$$
e^{-j 2 \pi\left(f_{2}-f_{1}\right) t}
$$

can be considered constant. The coherence time is thus in the order $1 /\left(f_{2}-f_{1}\right)$. By generalizing this two-path model, the coherence time can be approximately determined by $1 / \sigma_{v}$, where

$$
\sigma_{v} \triangleq \max _{i, i^{\prime}}\left(f_{i}-f_{i^{\prime}}\right)
$$

which is one measure of the Doppler spread of the channel. In symbolsampled time, the coherence time is thus approximately given by

$$
N_{\text {coherent }} \approx \frac{1}{T \sigma_{v}} .
$$


For example, in an outdoor environment, it can be assumed that the highest speed is $\left|v_{i}\right|<30 \mathrm{~m} / \mathrm{s}$ and that the maximum difference is $\max \left(v_{i}-v_{i^{\prime}}\right) \approx$ $60 \mathrm{~m} / \mathrm{s}$. In such an environment,

$$
\sigma_{v} \approx \frac{1}{\lambda} \max _{i, i^{\prime}}\left(v_{1}-v_{i^{\prime}}\right) \approx 400 \mathrm{~Hz},
$$

where $\lambda=0.15 \mathrm{~m}$ was assumed; that wavelength corresponds to an electromagnetic wave that propagates in air with the frequency $2 \mathrm{GHz}$. It is thus possible to track the channel for $N_{\text {coherent }}=750$ symbol durations if the baudrate is $300 \mathrm{kHz}$ and for 50000 symbol durations if the baudrate is $20 \mathrm{MHz}$.

In the study of a communication system, where the channel is evolving over time, it is sometimes assumed that the channel is block fading, i.e. that it is described accurately by the static impulse response $h[\ell]$ during one coherence time-for $N_{\text {coherent }}$ symbols. This block-fading model is used in all the included papers. A multipath channel model similar to (23) was used in Paper B. In the other papers, it is assumed that the channel is frequency selective and that the taps are Rayleigh fading, i.e. that in each coherence time the channel is equal to a realization of the random variable

$$
h[\ell] \sim \mathscr{C N N}\left(0, \sigma^{2}[\ell]\right),
$$

where $\sigma^{2}[\ell]$ is the power delay profile of the channel. Since the large-scale fading is given by $\beta$, the small-scale fading is normalized such that:

$$
\sum_{\ell=-\infty}^{\infty} \sigma^{2}[\ell]=1 .
$$

Because each path mainly contributes to only one channel tap in (23), it is also assumed that $\{h[\ell]\}$ is i.i.d. over $\ell$. Note that for $h[\ell]$ to be Gaussian, $\alpha_{i}^{2}$ should be Rayleigh distributed and $\left(f_{\mathrm{c}} \tau_{i}\right.$ mod 1$)$ uniformly distributed over $[0,1)$. If the number of paths is large however, the taps become Gaussian without any assumptions on the distributions of the individual paths because of the central limit theorem.

Another channel model used in the papers is the line-of-sight channel, in which it is assumed that there is only one path $N_{\mathrm{c}}=1$. This model can be a good approximation of the kind of channel that appears when the sight between transmitter and receiver is unobstructed and the signal is not reflected in the surrounding, for example, because of heavy signal attenuation, low 
reflectivity or because of the plain topology of the environment. The channel is then given by

$$
h(\tau)=\alpha_{1} e^{-j 2 \pi f_{\mathrm{c}} \tau_{1}} \delta\left(\tau-\tau_{1}\right) .
$$

With little loss in generality, it can be assumed that $\gamma\left(\ell T-\tau_{1}\right)=\delta[\ell]$ and that the discrete-time baseband channel is

$$
h[\ell]=\left\{\begin{array}{ll}
\alpha_{1} e^{-j 2 \pi f_{\mathrm{c}} \tau_{1}}, & \text { if } \ell=0 \\
0, & \text { otherwise }
\end{array} .\right.
$$

Bear in mind that the notion of coherence time that was defined in (36) makes little sense in a line-of-sight channel, where the a first order approximation of the evolution of the channel phase $f_{\mathrm{c}} \tau_{1}=d_{1}(0) / \lambda+f_{1} t$ many times makes it possible to accurately track the channel for a relatively long time.

In most environments, it is more realistic to believe that there is one or a few strong paths and many weaker paths, which is a situation in between Rayleigh fading and line-of-sight. In such a situation, the channel can be modeled as a weighted sum of a line-of-sight channel and a Rayleigh fading channel. The taps then follow a Rice distribution and the channel is said to be Ricean fading. In the included papers, only the Rayleigh fading and line-of-sight channels are studied. It is argued, as in [25], that if the results hold for both these cases, it is reasonable to believe that the results also hold approximately for a Ricean fading channel.

\subsection{Multi-User MIMO}

Massive MIMO is a multi-user MIMO system, which is a communication system where a base station that is equipped with $M$ antennas concurrently communicates with $K$ users. "Concurrently" here means that the signals to or from each user are sent at the same time and over the same frequency. The setup is shown in Figure 4, where each user has one antenna. Potentially, users can be equipped with multiple antennas to improve the data rate of the communication. One way to extend the analysis from single-antenna users to multi-antenna users is to treat each of the antennas of one user as if it were a user on its own. The actual rate of a user would then be the total rate of all its antennas. However, one should have in mind that the more antennas the power limited mobile user is equipped with, the bulkier and 


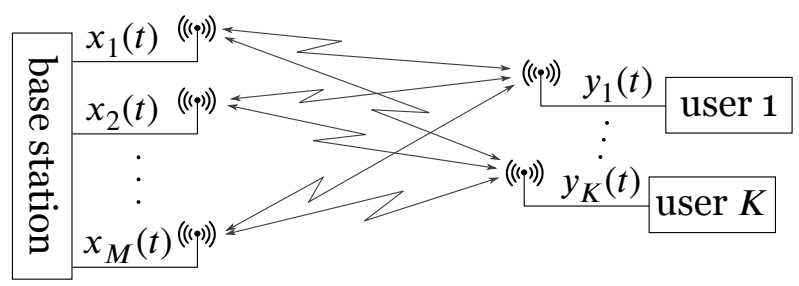

Figure 4: Multi-user MIMO with single-antenna users

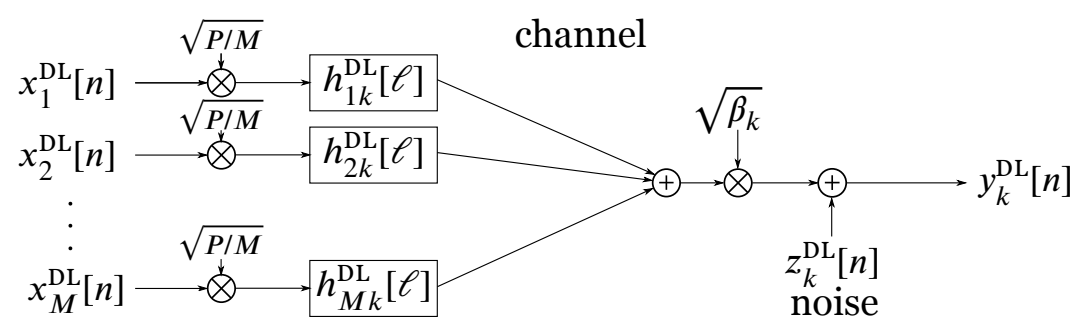

Figure 5: Discrete-time model of the downlink in a multi-user MIMO system

more power consuming its hardware becomes. For this reason, this thesis only studies users with single antennas. This is also a common assumption in the massive MIMo literature [26].

The transmission from the base station to the users is called the downlink and the reverse transmission, i.e. from the users to the base station, the uplink. Each channel between one base station antenna and one user can be analyzed in the same way as the fundamental communication system discussed in Section 2.1. The effect of having multiple signals concurrently transmitted in the downlink is that each user receives a sum of signals as shown in Figure 5 instead of only one signal as in Figure 2(b). If the channel between antenna $m$ and user $k$ is denoted $h_{m k}^{\mathrm{DL}}[\ell]$, then the received signal is

$$
y_{k}^{\mathrm{DL}}[n]=\sqrt{\beta_{k} P} \sum_{m=1}^{M} \sum_{\ell=-\infty}^{\infty} h_{m k}^{\mathrm{DL}}[\ell] x_{m}^{\mathrm{DL}}[n-\ell]+z_{k}^{\mathrm{DL}}[n],
$$

where $\beta_{k}$ is the large-scale fading attenuation to user $k, x_{m}^{\mathrm{DL}}[n]$ the transmit signal from antenna $m$ and $z_{k}^{\mathrm{DL}}[n]$ the thermal noise at user $k$. It is assumed that the base station antennas are co-located, so that all transmit signals are attenuated by the same large-scale fading coefficient $\beta_{k}$.

In the same way, the signal received by base station antenna $m$ in the uplink can be given in terms of the impulse response $h_{m k}^{\mathrm{UL}}[\ell]$ of the channel between 
user $k$ and the antenna in the following way:

$$
y_{m}^{\mathrm{UL}}[n]=\sum_{k=1}^{M} \sqrt{\beta_{k} P_{k}} \sum_{\ell=-\infty}^{\infty} h_{m k}^{\mathrm{UL}}[\ell] x_{k}^{\mathrm{UL}}[n-\ell]+z_{m}^{\mathrm{UL}}[n],
$$

where $x_{k}^{\mathrm{UL}}[n]$ and $P_{k}$ is the transmit signal and transmit power from user $k$ respectively and $z_{m}^{\mathrm{UL}}[n]$ is the thermal noise of the antenna.

The description of the downlink transmission can be simplified in the frequency domain by observing the transmission for a block of $N$ symbol durations and introducing a cyclic prefix, i.e. by observing $\left\{y_{k}^{\mathrm{DL}}[n], n=0, \ldots, N-1\right\}$ and letting

$$
x_{m}^{\mathrm{DL}}[n]=x_{m}^{\mathrm{DL}}[n+N], \quad n=-L+2, \ldots,-1
$$

in the downlink. The same can be done in the uplink. Then the time indices of the signals in the convolutions in (42) and (43) can be taken modulo $N$ and the convolutions can be seen as cyclic. The receive signal and channel are defined in the frequency domain as:

$$
\begin{aligned}
& y_{k}^{\mathrm{DL}}[v] \triangleq \frac{1}{\sqrt{N}} \sum_{n=0}^{N-1} y_{k}^{\mathrm{DL}}[n] e^{-j 2 \pi n v / N} \\
& h_{m k}^{\mathrm{DL}}[v] \triangleq \sum_{\ell=0}^{L-1} h_{m k}^{\mathrm{DL}}[\ell] e^{-j 2 \pi \ell v / N}
\end{aligned}
$$

Note that the frequency domain channel $h_{m k}^{\mathrm{DL}}[v]$ is not scaled by $1 / \sqrt{N}$, so that the received signals are given in the frequency domain as

$$
\begin{aligned}
& y_{k}^{\mathrm{DL}}[v]=\sqrt{P \beta_{k}} \sum_{m=1}^{M} h_{m k}^{\mathrm{DL}}[v] x_{m}^{\mathrm{DL}}[v]+z_{k}^{\mathrm{DL}}[v] \\
& y_{m}^{\mathrm{UL}}[v]=\sqrt{P_{k} \beta_{k}} \sum_{m=1}^{M} h_{m k}^{\mathrm{UL}}[v] x_{k}^{\mathrm{UL}}[v]+z_{m}^{\mathrm{UL}}[v] .
\end{aligned}
$$

The Fourier transforms $x_{m}^{\mathrm{DL}}[v], z_{k}^{\mathrm{DL}}[v], y_{m}^{\mathrm{UL}}[v], x_{k}^{\mathrm{UL}}[v]$ and $z_{m}^{\mathrm{UL}}[v]$ are defined analogous to $y_{k}^{\mathrm{DL}}[v]$ in (45); and $h_{m k}^{\mathrm{UL}}[v]$ to $h_{m k}^{\mathrm{DL}}[v]$ in (46).

\subsection{Channel Estimation}

For the massive MIMO base station to do coherent transmission and reception, it needs to estimate the down- and uplink channels. One way to do that is 
to dedicate part of the transmission to training signals, so called pilots. If the pilots are known to the receiver, they allow the receiver to estimate the channel over which the pilots have been transmitted. In massive MIMO, the uplink channel is estimated by letting the users transmit known $N_{\mathrm{p}}$-symbol long pilots $x_{k}^{\mathrm{UL}}[n]=\phi_{k}[n], n=0, \ldots, N_{\mathrm{p}}-1$. For notational simplicity, a dedicated transmission block for channel estimation is considered whose length is $N=N_{\mathrm{p}}$. The channel is also assumed to have support $L$, i.e. $h_{m k}^{\mathrm{UL}}[\ell]=0$ when $\ell \notin[0, \ldots, L-1], \forall m, k$. If the pilots are chosen such that:

$$
\sum_{n=0}^{N_{\mathrm{p}}-1} \phi_{k}[n] \phi_{k^{\prime}}^{*}[n+\ell]=\left\{\begin{array}{ll}
N_{\mathrm{p}}, & \text { if } k=k^{\prime}, \ell=0 \\
0, & \text { if } k \neq k^{\prime}, \ell=1, \ldots, L-1
\end{array},\right.
$$

where the indices are taken modulo $N_{\mathrm{p}}$, the base station can estimate the channel by correlating the received signal with the known pilot sequences:

$$
r_{m k}[\ell]=\sum_{n=0}^{N_{\mathrm{p}}-1} x_{m}^{\mathrm{UL}}[n] \phi_{k}^{*}[n+\ell] .
$$

By using (43) and (49), it can be seen that

$$
r_{m k}[\ell]=\sqrt{\beta_{k} P_{k}} N_{\mathrm{p}} h_{m k}[\ell]+z_{m k}^{\prime}[\ell],
$$

where

$$
z_{m k}^{\prime}[\ell] \triangleq \sum_{n=0}^{N_{\mathrm{p}}-1} z_{m}^{\mathrm{UL}}[n] \phi_{k}^{*}[n+\ell] \sim \mathscr{C} \mathcal{N}\left(0, \frac{N_{\mathrm{p}} N_{0}}{T}\right)
$$

is a white Gaussian noise sequence that is uncorrelated across $\ell$. The correlation output $r_{m k}[\ell]$ is thus a noisy observation of the channel coefficient $h_{m k}^{\mathrm{UL}}[\ell]$. Note that the length of the pilot has to be $N_{\mathrm{p}} \geq K L$ in order to fulfill (49). If we assume that the base station knows the power delay profile of the channel, i.e. the powers

$$
\sigma_{m k}^{2}[\ell] \triangleq \mathrm{E}\left[\left|h_{m k}^{\mathrm{UL}}[\ell]\right|^{2}\right],
$$

then the linear minimum mean-square-error (LMMSE) estimate of the channel is obtained by

$$
\begin{aligned}
\hat{h}_{m k}^{\mathrm{UL}}[\ell] & =\frac{\mathrm{E}\left[h_{m k}^{\mathrm{UL}}[\ell] r_{m k}^{*}[\ell]\right]}{\mathrm{E}\left[\left|r_{m k}[\ell]\right|^{2}\right]} r_{m k}[\ell] \\
& =\frac{\sqrt{\beta_{k} P_{k}} \sigma_{m k}^{2}[\ell]}{\beta_{k} P_{k} N_{\mathrm{p}} \sigma_{m k}^{2}[\ell]+N_{0} / T} r_{m k}[\ell]
\end{aligned}
$$


and its mean-square-error is given by

$$
\begin{aligned}
\mathrm{E}\left[\left|\hat{h}_{m k}^{\mathrm{UL}}[\ell]-h_{m k}^{\mathrm{UL}}[\ell]\right|^{2}\right] & =\sigma_{m k}^{2}[\ell]-\frac{\left|\mathrm{E}\left[h_{m k}^{\mathrm{UL}}[\ell] r_{m k}^{*}[\ell]\right]\right|^{2}}{\mathrm{E}\left[\left|r_{m k}[\ell]\right|^{2}\right]} \\
& =\sigma_{m k}^{2}[\ell]\left(1-c_{m k}[\ell]\right),
\end{aligned}
$$

where

$$
c_{m k}[\ell] \triangleq \frac{\beta_{k} P_{k} N_{\mathrm{p}} \sigma_{m k}^{2}[\ell]}{\beta_{k} P_{k} N_{\mathrm{p}} \sigma_{m k}^{2}[\ell]+N_{0} / T} .
$$

The LMMSE estimate of the frequency-domain channel is given by

$$
\hat{h}_{m k}^{\mathrm{UL}}[\nu] \triangleq \sum_{\ell=0}^{L-1} \hat{h}_{m k}^{\mathrm{UL}}[\ell] e^{-j 2 \pi \ell v / N} .
$$

The mean-square error of the frequency-domain channel estimate is

$$
\mathrm{E}\left[\left|\hat{h}_{m k}^{\mathrm{UL}}[\ell]-h_{m k}^{\mathrm{UL}}[\ell]\right|^{2}\right]=1-c_{m k},
$$

where the channel estimation quality is defined as

$$
c_{m k} \triangleq \sum_{\ell=0}^{L-1} \sigma_{m k}^{2}[\ell] c_{m k}[\ell]=\sum_{\ell=0}^{L-1} \frac{\sigma_{m k}^{4}[\ell] \beta_{k} P_{k} N_{\mathrm{p}}}{\sigma_{m k}^{2}[\ell] \beta_{k} P_{k} N_{\mathrm{p}}+N_{0} / T} .
$$

Remember that the large-scale fading $\beta_{k}$ of the downlink and uplink channels were the same in (42) and (43). This is a very natural assumption: the uplink and downlink signals travel the same paths, just in opposite directions, and should experience the same attenuation. If the small-scale fading is reciprocal in the same way, i.e. that $h_{m k}^{\mathrm{DL}}[\ell]=h_{m k}^{\mathrm{UL}}[\ell]$, then it is enough to estimate the uplink channel to also get an estimate of the downlink channel. If the transmission is done in so-called time-division duplex (TDD), i.e. the uplink and downlink use the same frequency band and are separated in time, it makes sense to assume that the channel is reciprocal as long as the time separation is short. In practice, the hardware is not reciprocal and requires calibration to avoid mismatches between the uplink and downlink when exploiting channel reciprocity. Since this hardware mismatch changes slowly over time, it can be handled by relatively simple calibration methods [6]. Massive MIMO is usually assumed to be implemented in TDD as in Figure 6 in order to use the reciprocity argument to avoid estimating the downlink 


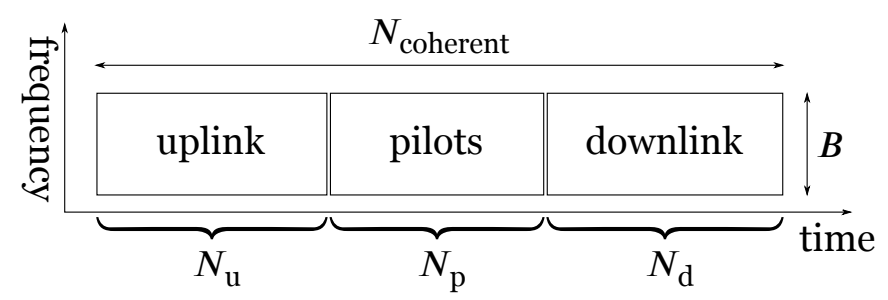

Figure 6: Massive MIMO frame structure

channel directly. In TDD mode, the whole allocated bandwidth is first used for the uplink, where the users transmit a block of $N=N_{\mathrm{u}}$ symbols, and then for the training period ${ }^{2}$, where the users transmit a block of $N=N_{\mathrm{p}}$ pilots. Thereafter, the whole bandwidth is used for the downlink and the base station transmits a block of $N=N_{\mathrm{d}}$ symbols to each user.

In this thesis, TDD transmission and full reciprocity is assumed. The superscript in the notation is therefore dropped

$$
h_{m k}[\ell]=h_{m k}^{\mathrm{UL}}[\ell]=h_{m k}^{\mathrm{DL}}[\ell] .
$$

Similarly, the channel estimate is written:

$$
\hat{h}_{m k}[\ell] \triangleq \hat{h}_{m k}^{\mathrm{UL}}[\ell],
$$

which thus also is the estimate of the downlink channel. In analogy to the time-domain notation, the frequency-domain channel is denoted $h_{m k}[v]$ and its estimate $\hat{h}_{m k}[v]$. Note that these Fourier transforms are implicit functions of the block size $N$, which not necessary is the same in the up- and downlink.

To estimate the actual downlink channel, each base station antenna would have to transmit pilots that fulfill a constraint similar to (49) (the indices would run from 1 to $M$ instead of to $K$ ). These pilots therefore would have to be at least $M L$ symbols long. Since it would be the users who estimate the channel, they would have to feed the estimate back to the base station. Both the $M L$-symbol long downlink pilots and the feedback would consume valuable resources that otherwise could be used for data transmission. This is one reason for wanting to avoid actual estimation of the downlink channel. Another reason is to let the number of antennas be fully scalable, i.e. the

\footnotetext{
${ }^{2}$ It is not strictly required that the uplink data transmission and the training period are separate in time. The users could send pilots interleaved with the uplink data. Treating the uplink and the training period as separate blocks does not compromise the generality of the results however, and is a way to simplify the mathematical exposition.
} 
system design should be such that the number of antennas can be increased without limit. If $M$ can be arbitrary large, then $M L$ long pilots would no longer fit in a finite coherence time.

The number of symbol durations that have to be used for channel estimation is thus $N_{\mathrm{p}}=\mu K L$, where a pilot excess factor $\mu=1$ gives the shortest possible pilots that still fulfill (49). Since the channel has to be estimated once every coherence time, i.e. once every $N_{\text {coherent }}$ symbol durations, one has to consider if the length of the pilots fits in this interval and how much of the coherence time that has to be used for channel estimation and not for data transmission. Using the relations in (25) and (36), the fraction of the coherence time that is used for channel estimation is

$$
\begin{aligned}
\frac{N_{\mathrm{p}}}{N_{\text {coherent }}} & \approx \frac{\mu K \sigma_{\tau} / T}{1 /\left(T \sigma_{v}\right)} \\
& =\mu K \sigma_{\tau} \sigma_{v} .
\end{aligned}
$$

For this fraction to be less than one, the number of users has to be limited to below

$$
K<\frac{1}{\mu \sigma_{\tau} \sigma_{v}} .
$$

The coherence time will therefore limit the number of user that can be served, but not the number of antennas the base station can be equipped with. Using the example numbers derived for the outdoor channel in Section $2.2\left(\sigma_{\tau}=\right.$ $3.3 \mu$ s and $\sigma_{v}=400 \mathrm{~Hz}$ ), we get

$$
\begin{aligned}
\frac{N_{\mathrm{p}}}{N_{\text {coherent }}} & \approx 0.00132 \mu K, \\
K & <\frac{757}{\mu} .
\end{aligned}
$$

Since the number of active users in one communication system seldom is as large as 757, this indicates that this kind of pilot-based channel estimation is feasible with regards to the coherence time as long the pilot excess factor is close to one.

In a single-cell system, where one base station serves a set of users without being disturbed by neighboring transmission, a pilot excess factor of one is often enough to obtain good channel estimates. The reason is that the total received energy of the observations in (51) increases with the pilot length, 
which is large in a multiuser system also with $\mu=1$. A pilot excess factor greater than one might be needed in a multicell system, however, where many base stations concurrently serve disjoint sets of users. If transmission from other cells is present on the same frequency and at the same time that the pilots are sent, then pilot contamiation arises [1], i.e. the channel estimates obtained are contaminated by the nonorthogonal interfering signals from the other cells. Apart from decreasing the channel estimation quality, pilot contamination also increases the intercell interference. One way to avoid pilot contamination is to synchronize the channel estimation between all cells using the same frequency and to make each pilot orthogonal to all the other pilots in all cells. This would require a pilot excess factor larger than one. Other techniques have been proposed to mitigate pilot contamination [27,28]. The fact, however, remains: in practice, the finite coherence time of the channel constrains the length of the pilots and thus the number of users that can be served in each cell. Luckily, the maximum number of users is large in many systems, as was seen in the example (68).

\subsection{Precoding and Combining}

To send information, the base station precodes the signals in such a way that each user receives the signal that is intended for it. To receive information that each user has transmitted, the base station combines the received signals in such a way that the information from each user is separated.

In the downlink, the symbols that are to be transmitted to the user $k$ are denoted $s_{k}^{\mathrm{DL}}[v], v=0, \ldots, N_{\mathrm{d}}-1$. A linear precoder weights these symbols by the precoding weights $w_{m k}[v]$ to produce the transmit signal:

$$
x_{m}^{\mathrm{DL}}[v]=\sum_{k=1}^{K} w_{m k}[v] \sqrt{\xi_{k}} s_{k}^{\mathrm{DL}}[v],
$$

where the power allocation factors $\xi_{k}$ make it possible to allocate power between the users. To ensure that the energy of the transmit signal meets the power constraint:

$$
\sum_{m=1}^{M} \mathrm{E}\left[\left|x_{m}^{\mathrm{DL}}[v]\right|^{2}\right]=1, \quad \forall v,
$$


the power allocation factors sum to unity: $\sum \xi_{k}=1$, the symbols are normalized to unit power: $\mathrm{E}\left[\left|s_{k}^{\mathrm{DL}}[v]\right|^{2}\right]=1$, and the precoding weights are scaled such that

$$
\sum_{k=1}^{K} \sum_{m=1}^{M} \xi_{k} \mathrm{E}\left[\left|w_{m k}[v]\right|^{2}\right]=K, \quad \forall v .
$$

For mathematical tractability, the expectations in (70) and (71) are taken with respect also to the small-scale fading of the channel in this thesis. This is called a long-term power constraint, because it ensures that the radiated power meets the constraint when averaged over many coherence times. In a practical system, it might be desirable to fulfill the power constraint in each coherence time and take the expectation in (70) with respect to the random symbols and remove the expectation operator in (71). This is called a shortterm power constraint. Due to the law of large numbers, however, long-term and short-term power constraints are very similar in massive MIMO when the channel to different users are uncorrelated. Therefore the choice of power constraint makes little difference to the system performance.

To see the effect of the precoding on the received signal, write the channel as the sum of the estimated channel and the estimation error: $h_{m k}[v]=$ $\hat{h}_{m k}[v]+e_{m k}[v]$. Then the received signal of user $k$ is given by:

$$
\begin{aligned}
y_{k}^{\mathrm{DL}}[v]= & \sqrt{\beta_{k} P \xi_{k}} s_{k}^{\mathrm{DL}}[v] \sum_{m=1}^{M} \hat{h}_{m k}[v] w_{m k}[v] \\
& +\sqrt{\beta_{k} P} \sum_{k^{\prime} \neq k} \sqrt{\xi_{k^{\prime}}} s_{k^{\prime}}^{\mathrm{DL}}[v] \sum_{m=1}^{M} \hat{h}_{m k}[v] w_{m k^{\prime}}[v] \\
& +\sqrt{\beta_{k} P} \sum_{k^{\prime}=1}^{K} \sqrt{\xi_{k^{\prime}}} s_{k^{\prime}}^{\mathrm{DL}[v]} \sum_{m=1}^{M} e_{m k}[v] w_{m k^{\prime}}[v]+z_{k^{\prime}}^{\mathrm{DL}}[v]
\end{aligned}
$$

The received signal is thus the sum of four terms: one desired signal that contains the symbol $s_{k}^{\mathrm{DL}}[v]$, one interuser interference that contains the symbols to other users $s_{k^{\prime}}^{\mathrm{DL}}[v]$, one term that contains the channel estimation error and one noise term. By choosing the weights properly, it is possible to make the desired term large and to suppress the interuser interference term; without knowledge of the channel estimation error, little can be done about the estimation error term nor the noise term. As will be seen later however, these terms combine noncoherently and their power becomes small in comparison to the power of the desired signal. With proper precoding, the 
desired signal will thus dominate and the users can treat the other terms as noise. They never have to do any advanced equalization and therefore do not need to estimate the channel. They use the received signal directly as their symbol estimate:

$$
\hat{s}_{k}^{\mathrm{DL}}[v] \triangleq y_{k}^{\mathrm{DL}}[v]
$$

Other nonlinear precoders, which map the symbols onto the transmit signals in a nonlinear fashion, include the discrete-time constant-envelope precoder that is studied in Paper A and the continuous-time constant-envelope precoder that is proposed in Paper B. Both of these precoders aim at producing precoded signals that are easy to generate with low-end hardware.

In the uplink, the base station performs combining instead of precoding. Again, the users do not need any knowledge of the channel nor do they have to do any special signal processing. They just transmit their symbols $s_{k}^{\mathrm{UL}}[v]$ in the frequency domain, i.e. let $x_{k}^{\mathrm{UL}}[v]=s_{k}^{\mathrm{UL}}[v]$. To meet the power constraint, the symbols are normalized to have unit power: $\mathrm{E}\left[\left|s_{k}^{\mathrm{UL}}[v]\right|^{2}\right]=1$. The base station then applies a linear combiner to the received signal to obtain the symbol estimate:

$$
\hat{s}_{k}^{\mathrm{UL}}[v] \triangleq \sum_{m=1}^{M} w_{m k}[v] y_{m}^{\mathrm{UL}}[v] .
$$

Note that the combiner weights and the precoding weights are the same, since they are equalizing the same channel. The output of the combiner is an estimate of the transmitted symbol.

Described above was an orthogonal frequency-division multiplexing (OFDM) based transmission, where the symbols were transmitted in the frequency domain on different subcarriers. Massive MIMO can also be operated with single-carrier transmission. Then the symbols $s_{k}^{\mathrm{DL}}[n]$ that are transmitted in the downlink are Fourier transformed into:

$$
s_{k}^{\mathrm{DL}}[v]=\frac{1}{\sqrt{N_{\mathrm{d}}}} \sum_{n=0}^{N_{\mathrm{d}}-1} s_{k}^{\mathrm{DL}}[n] e^{-j 2 \pi n v / N_{\mathrm{d}}}
$$

and the frequency-domain symbols $s_{k}^{\mathrm{DL}}[v]$ are precoded, i.e. the transmit signal is obtained by substituting $s_{k}^{\mathrm{DL}}[v]$ by $s_{k}^{\mathrm{DL}}[v]$ in (69). The users obtain their symbol estimates in the time domain as $\hat{s}_{k}^{\mathrm{DL}}[n]=y_{k}[n]$ instead of in the 
frequency domain as in (73). Similarly for the uplink, the users transmit the symbols directly in the time domain: $y_{k}[n]=s_{k}^{\mathrm{UL}}[n]$ and the base station obtains the symbol estimates by performing combining as in (74) and inverse transforming the output:

$$
\hat{s}_{k}^{\mathrm{UL}}[n]=\frac{1}{\sqrt{N_{\mathrm{d}}}} \sum_{\nu=0}^{N_{\mathrm{d}}-1} \sum_{m=1}^{M} w_{m k}[v] y_{m}^{\mathrm{UL}}[v] e^{j 2 \pi n v / N_{\mathrm{d}}}
$$

instead of using the frequency domain signal as in (74). Note that the users never have to perform any Fourier transforms with single-carrier transmission, the base station performs the Fourier transforms instead.

In Paper A, the two transmission methods, OFDM and single-carrier transmission, are compared. It is found that the PAR of the downlink transmit signal, and thus the hardware distortion, in single-carrier and OFDM systems are almost identical in massive MIMO, and in many cases, e.g. when the channel has a long delay spread, they are identical. This stands in contrast to classical systems, where single-carrier transmission results in signals with significantly lower PAR than OFDM transmission. Furthermore, it is observed that single-carrier and OFDM transmission result in similar computational complexity. Single-carrier transmission can be implemented with FIR filters with short delays, whereas OFDM requires the precoding to be done in blocks, which results in longer delays. On the other hand, OFDM allows for scheduling in the frequency domain. In short, the two transmission methods are very similar in many aspects in massive MIMO and which is to prefer depends on the application and many times it seems not to matter which one is used.

The performance of the precoder and combiner can be evaluated in terms of an achievable rate, i.e. the amount of information that can be reliably transmitted on average during one symbol duration. To allow for a compact exposition, both the up- and downlink symbols $s_{k}^{\mathrm{UL}}[n], s_{k}^{\mathrm{DL}}[n]$ are denoted by $s_{k}[n]$, and their estimates $\hat{s}_{k}^{\mathrm{UL}}[n], \hat{s}_{k}^{\mathrm{DL}}[n]$ by $\hat{s}_{k}[n]$ in the following achievable rate:

$$
R_{k}=\log _{2}\left(1+\frac{\left|\mathrm{E}\left[s_{k}^{*}[n] \hat{s}_{k}[n]\right]\right|^{2}}{\mathrm{E}\left[\left|\hat{s}_{k}[n]\right|^{2}\right]-\left|\mathrm{E}\left[s_{k}^{*}[n] \hat{s}_{k}[n]\right]\right|^{2}}\right) \quad\left[\mathrm{bpcu}^{3}\right] .
$$

By using Gaussian distributed symbols, the expectations can be evaluated and an achievable rate is obtained. The rate can be derived based on results

${ }^{3}$ Bits-Per-Channel-Use, the amount of information that can be conveyed on average during the symbol time $T$ over the bandwidth $B$ measured in bits. 
in [29] as in Paper D. This achievable rate can be used to evaluate the performance of any precoder and combiner, also nonlinear ones such as the low-PAR precoders. The expectations in (77) should be conditioned on the channel state information available at the receiver. Since the users only know the large-scale fading, the expectations are evaluated with respect to the small-scale fading in the downlink. Thus, it is assumed that the information is coded over many realizations of the small-scale fading-over many coherence intervals. In the uplink, the base station has an estimate of the channel. It would therefore be possible to condition the expectations on the channel estimate to obtain a higher rate. To derive an expression that does not depend on the channel, however, it will be assumed that the base station "forgets" its channel state information after the combining. Hence, the expectations are evaluated without any conditioning also for the uplink. The thus obtained achievable rate is sometimes referred to as the use-and-forget bound.

For linear precoders and combiners, the rate in (77) can be further analyzed if we assume that the power delay profile is the same for all antennas, i.e. $\sigma_{m k}^{2}[\ell]=\sigma_{k}^{2}[\ell]$. Then $c_{m k}=c_{k}$ for all antennas $m$ for some common channel estimation quality $c_{k}$. It can be seen from (72) and (73) that the numerator in (77) equals $c_{k} \xi_{k} \beta_{k} P G_{k}$ in the downlink, where the array gain is defined as

$$
G_{k} \triangleq \frac{1}{c_{k}}\left|\mathrm{E}\left[\sum_{m=1}^{M} w_{m k}[v] \hat{h}_{m k}[v]\right]\right|^{2} .
$$

Similarly, the numerator equals $c_{k} \beta_{k} P_{k} G_{k}$ in the uplink. The downlink channel in (72) and (73) can thus be rewritten as a SISO link with a deterministic effective channel gain:

$$
\begin{aligned}
\hat{s}_{k}^{\mathrm{DL}}[v]= & \sqrt{\beta_{k} P \xi_{k}} s_{k}^{\mathrm{DL}}[v] \mathrm{E}\left[\sum_{m=1}^{M} w_{m k}[v] \hat{h}_{m k}[v]\right] \\
& +\sqrt{\beta_{k} P} \sum_{k^{\prime}=1}^{K} \sqrt{\xi_{k^{\prime}}} s_{k^{\prime}}^{\mathrm{DL}}[v]\left(\sum_{m=1}^{M} w_{m k^{\prime}}[v] \hat{h}_{m k}[v]-\delta\left[k-k^{\prime}\right] \mathrm{E}\left[\sum_{m=1}^{M} w_{m k^{\prime}}[v] \hat{h}_{m k}[v]\right]\right) \\
& +\sqrt{\beta_{k} P} \sum_{k^{\prime}=1}^{K} \sqrt{\xi_{k^{\prime}}} s_{k^{\prime}}^{\mathrm{DL}}[v] \sum_{m=1}^{M} e_{m k}[v] w_{m k^{\prime}}[v]+z_{k^{\prime}}^{\mathrm{DL}}[v]
\end{aligned}
$$

where $\delta[n]=1$ when $n=0$ otherwise $\delta[n]=0$. The second term is the interuser interference from user $k^{\prime}$ onto user $k$; its normalized variance is denoted

$$
I_{k k^{\prime}} \triangleq \frac{1}{c_{k}} \mathrm{E}\left[\left|\sum_{m=1}^{M} w_{m k^{\prime}}[v] \hat{h}_{m k}[v]-\delta\left[k-k^{\prime}\right] \mathrm{E}\left[\sum_{m=1}^{M} w_{m k^{\prime}}[v] \hat{h}_{m k}[v]\right]\right|^{2}\right]
$$


in the downlink and $I_{k^{\prime} k}$ in the uplink. It is observed that the self-interference $I_{k k}$ generally is non-zero. In the downlink, this is natural because the user, who only has statistical knowledge about the channel, does not know the actual effective channel gain of the transmission $\sum_{m=1}^{M} w_{m k}[v] \hat{h}_{m k}[\nu]$. By using its expected value as the estimate as in (79), an estimation error arises, whose variance is captured by the self-interference $I_{k k}$. In many massive MIMO channels, this simplistic estimation of the effective channel gain works well, but more sophisticated estimation methods of the effective channel gain have been proposed [30]. Arguably, the self-interference should be zero in the uplink, because the base station has channel state information and can compute the effective channel gain. Using $I_{k k}=0$ would yield a higher rate than what is obtained from the interference in (80). The improvement is small however and the definition in (80) is used in this thesis also for $k^{\prime}=k$ for simplicity.

Since the power of the third term, the error due to imperfect channel state information, is $K \beta_{k} P\left(1-c_{k}\right)$ in the downlink and $\sum_{k^{\prime}=1}^{K} \beta_{k^{\prime}} P_{k^{\prime}}\left(1-c_{k^{\prime}}\right)$ in the uplink, the rate in (77) becomes

$$
R_{k}=\log _{2}\left(1+\frac{c_{k} \xi_{k} \beta_{k} P G_{k}}{\beta_{k} P \sum_{k^{\prime}=1}^{K}\left(1-c_{k}\left(1-\xi_{k^{\prime}} I_{k k^{\prime}}\right)\right)+N_{0} / T}\right) \quad[\mathrm{bpcu}]
$$

for linear precoders in the downlink and

$$
R_{k}=\log _{2}\left(1+\frac{c_{k} \beta_{k} P_{k} G_{k}}{\sum_{k^{\prime}=1}^{K} \beta_{k^{\prime}} P_{k^{\prime}}\left(1-c_{k^{\prime}}\left(1-I_{k^{\prime} k}\right)\right)+N_{0} / T}\right) \quad[\mathrm{bpcu}]
$$

for linear combiners in the uplink. Because the array gain and interference suppression properties of a precoder and combiner are captured by the two parameters $G_{k}$ and $I_{k^{\prime} k}$, they are referred to as the characteristic parameters of the signal processing method.

The precoder and combiner thus determines how large the array gain $G_{k}$ and how small the interference $I_{k k^{\prime}}$ is, and there is a trade-off between the two. The precoder and combiner that maximizes the array gain is the maximumratio precoder and combiner, which is given by

$$
w_{m k}[v]=\frac{1}{\sqrt{c_{k} M}} \hat{h}_{m k}^{*}[v] .
$$


Interference, however, is not suppressed. If the channel is i.i.d. Rayleigh fading, i.e. if $h_{m k}[\ell] \sim \mathscr{C} \mathcal{N}\left(0, \sigma_{k}^{2}[\ell]\right)$, the characteristic parameters of the maximum-ratio precoder and combiner are:

$$
G_{k}=M, \quad I_{k k^{\prime}}=1 .
$$

The precoder and combiner that minimizes the interference power is the zero-forcing precoder and combiner, which has to be defined in terms of the estimated channel matrix

$$
\hat{\mathbf{H}}[v] \triangleq\left(\begin{array}{ccc}
\hat{h}_{11}[v] & \cdots & \hat{h}_{1 K}[v] \\
\vdots & \ddots & \vdots \\
\hat{h}_{M 1}[v] & \cdots & \hat{h}_{M K}[v]
\end{array}\right)
$$

and its pseudo-inverse

$$
\mathbf{M}_{0}[v]=\hat{\mathbf{H}}^{*}[v]\left(\hat{\mathbf{H}}^{\top}[v] \hat{\mathbf{H}}^{*}[v]\right)^{-1} .
$$

If $\left[\mathbf{M}_{0}[v]\right]_{m k}$ is the $(m, k)$-th element of the pseudo-inverse $\mathbf{M}_{0}[v]$, the zeroforcing weights are given by

$$
w_{m k}[v]=\sqrt{(M-K) c_{k}}\left[\mathbf{M}_{0}[v]\right]_{m k} .
$$

If the channel is i.i.d. Rayleigh fading, the characteristic parameters for the zero-forcing precoder and combiner are:

$$
G_{k}=M-K, \quad I_{k k^{\prime}}=0 .
$$

A choice that enables us to balance the array-gain maximization and interference suppression is the $\lambda$-regularized zero-forcing precoder and combiner, which is given in terms of the matrix

$$
\mathbf{M}_{\lambda}[v]=\hat{\mathbf{H}}^{*}[v]\left(\hat{\mathbf{H}}^{\top}[v] \hat{\mathbf{H}}^{*}[v]+\lambda \mathbf{I}_{K}\right)^{-1} .
$$

The regularized zero-forcing weights are given by

$$
w_{m k}[v]=a\left[\mathbf{M}_{\lambda}[v]\right]_{m k},
$$

where $a$ is some normalizing factor that has to be chosen to fulfill the power constraint in (71). Note that a large regularizing factor $\lambda$ results in a precoder similar to the maximum-ratio precoder, and a small $\lambda$ in a precoder similar to the zero-forcing precoder. In general, the regularizing term $\lambda \mathbf{I}_{k}$ would be a diagonal matrix with $K$ regularizing diagonal terms that not necessarily are the same. It was shown in [31] that in massive MIMO, using the same 
regularizing factor on the diagonal is close to optimal if the aim is to give all users the same rate. This brings down the dimension of the parameter space to one and simplifies the implementation of the regularized zero-forcer.

Using (81), (82) and the values of $G_{k}$ and $I_{k^{\prime} k}$, closed-form achievable rates for maximum-ratio and zero-forcing precoding are obtained. These expressions were first derived for a flat-fading channel in [32] and then extended to frequency-selective channels for maximum-ratio precoding in [33]. These previous data rates are special cases of the more general rates in (81) and (82) that are derived in Paper A for the frequency-selective downlink with nonlinear power amplifiers and in Paper D for the frequency-selective uplink with one-bit ADCs. The achievable rate in (77) is also used in Paper B. 


\section{Chapter 3}

\section{Hardware}

The hardware that generates a transmit signal from the discrete-time complex baseband signal is called a transmitter. The corresponding hardware that converts the received passband signal into a discrete-time complex baseband signal is called a receiver. Below follows a brief introduction to the hardware of the transmit and receivers of the massive MIMO base station.

\subsection{Transmitter Design}

First the conventional transmitter in Figure 7(a) is studied. The complexity, power consumption and cost of this conventional transmitter, however, makes it less attractive to use in massive MIMO, which requires hundreds of transmitters-one for each antenna. For massive MIMO, instead, it is more relevant to study a low-end transmitter that employs inexpensive hardware and that excludes much of the more complex hardware, which is not absolutely necessary in the signal generation. An example of such a low-end design is shown in Figure 7(b), which is the design studied in the included Papers A, B and C.

\subsubsection{Upsampling}

Both with single-carrier and OFDM transmission, the first step in the transmitter is to upsample the symbol-sampled baseband signal $x[n]$ to produce 


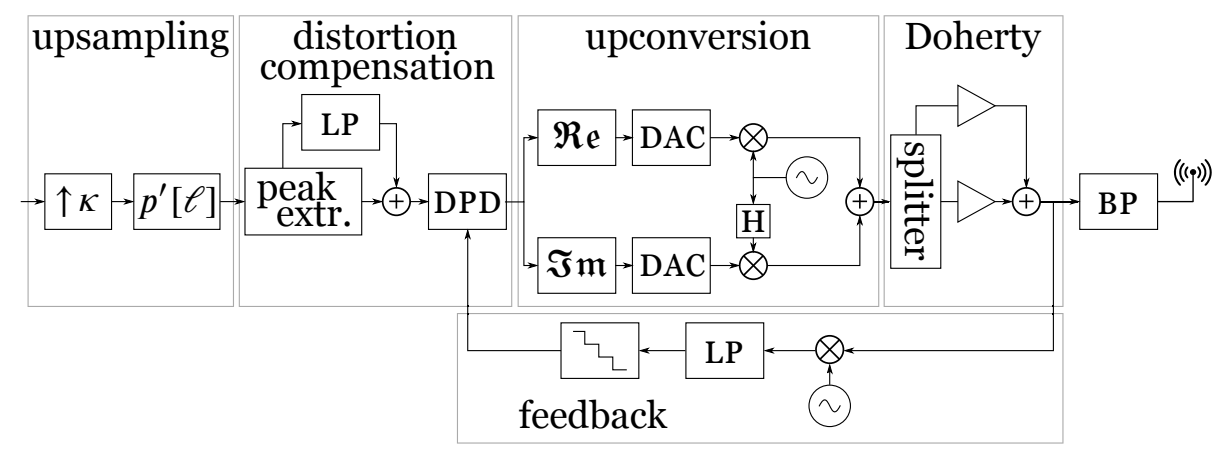

(a) Conventional design

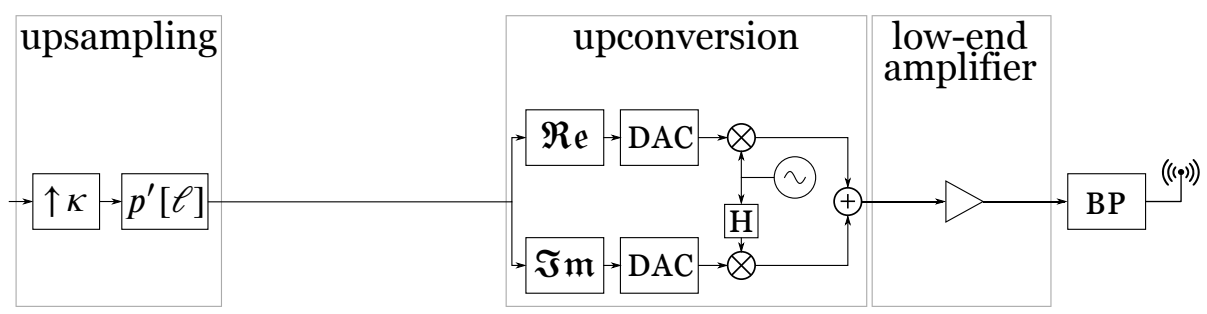

(b) Low-end design

Figure 7: A general transmitter design

the $\kappa$-times oversampled signal $x^{(\kappa)}[n]$. This is done by zero-stuffing, i.e. by inserting $\kappa-1$ zero-samples between each original sample to produce

$$
x_{\mathrm{zS}}[n] \triangleq \begin{cases}x[n / \kappa], & n \equiv 0 \bmod K \\ 0, & \text { otherwise }\end{cases}
$$

The zero-stuffed signal is then pulse-shape filtered with the pulse $p^{\prime}[\ell] \triangleq$ $p^{\prime}(\ell T / \kappa)$, which is the discrete-time equivalent of the filter $p^{\prime}(\tau)$ discussed in Section 2.1. The resulting upsampled signal is

$$
x^{(\kappa)}[n]=\sum_{\ell=-\infty}^{\infty} p^{\prime}[\ell] x_{\mathrm{zS}}[n-\ell] .
$$

\subsubsection{Distortion Compensation}

The upsampled baseband signal is then preprocessed to compensate for the imperfections of the analog components, such as the oscillators, mixers and power amplifiers. Two compensation techniques commonly used in 
association with the power amplifiers are: crest-factor reduction that lowers the PAR of the signal to enable amplification with higher power efficiency, and digital predistortion (DPD) that tries to linearize the combined transfer function of the DPD and the nonlinear power amplifier to avoid nonlinear signal distortion.

Crest-factor reduction can be done in many ways, e.g., repeated clipping-andfiltering, tone reservation and peak cancellation, see [34,35] for an overview of crest-factor reduction techniques. Some of the techniques are specific for OFDM, such as tone reservation, others can be used independently of the transmission method, such as clipping-and-filtering. Sometimes several techniques are used together to reduce the PAR further. Different crestfactor reduction techniques, however, have different drawbacks. Some cause distortion to the in-band signal, i.e. distortion that has frequency components inside the frequency band of the original signal, e.g., clipping-and-filtering. Some cause delays to the signal, e.g., clipping-and-filtering, peak cancellation. And some have to use some of the degrees-of-freedom of the signal that otherwise could have been used for data transmission, which reduces the effective data rate of the system, e.g., tone reservation and peak-cancellation that uses reserved frequencies.

The DPD distorts the signal in such a way that the output of the power amplifier will be as close to what the output would have been had the amplifier been perfectly linear. To know how to do this compensation, the output of the amplifier has to be observed, which is made possible by the feedback loop in Figure $7(\mathrm{a})$. Since the amplified signal is an analog passband signal, it has to be downconverted and digitalized in an ADC before being fed back to the DPD, which adds to the hardware complexity.

To build a massive MIMO base station, one would need hundreds of transmitters. Functionality, such as the DPD, that consumes a fixed amount of power independently of the output power does not allow for scalability in the number of base station antennas and becomes impractical in a massive MIMO base station. Other components, such as crest-factor reduction, have a complexity that is affordable in systems with a few transmitters. In massive MIMO however, their complexity becomes high and might not be feasible. For this reason, the low-end transmitter in Figure $7(\mathrm{~b})$ that is studied in this thesis has no distortion compensation nor a feedback loop. Crest-factor reduction can still be done on the symbol-sampled baseband signal by applying a low-PAR precoding method, as the ones studied in Paper A. 


\subsubsection{Upconversion}

To obtain a passband signal as in (9), the complex baseband signal is split up in its real and imaginary parts, each of which are converted into an analog signal by a digital-to-analog converter (DAC). These real baseband signals are upconverted to passband by mixing them with their carrier waves. The carrier wave for the real part is obtained directly from an oscillator that operates at the carrier frequency $f_{\mathrm{c}}$. The carrier wave for the imaginary part is a copy of the oscillator output that is delayed by $90^{\circ}$. The delay is denoted by $\mathrm{H}$ in Figure 7, where $\mathrm{H}$ stands for Hilbert transform. The passband signal is obtained by summing the two signals up.

\subsubsection{Amplification}

The passband signal is then amplified to transmit power. Often, in base stations with transmit signals with high PAR, the amplification is done by multi-transistor amplifiers such as the Doherty amplifier [36]. In the simplest Doherty amplifier, the signal is split up and amplified by two separate amplifiers and then summed up. The Doherty amplifier gives a more linear amplification and a high power efficiency. For a detailed description of the Doherty amplifier, see [37, Ch. 9]. The splitting and summation are difficult to implement perfectly, which can result in distortion. This is traditionally handled by the DPD or by doing the splitting in the digital domain, which would require the splitter to be moved to the front of the upconvertion stage and each amplifier would need its own upconvertion stage-a so called digital dual-input Doherty.

In massive MIMO, where each antenna would need its own amplifier, only simple amplifier designs would be feasible, such as class A, B or AB amplifiers. If the splitting and summing in the Doherty amplifier can be done in the analog domain without a DPD, massive MIMO could possibly also be built with simple single-input analog Doherty amplifiers.

The memoryless nonlinearity of an amplifier can be illustrated by its transfer characteristics, an example of which is shown in Figure 8. The transfer characteristics show what amplitude the output signal will have as a function of the amplitude of the input signal. If the amplifier were linear, the curve would be linear with unity slope. The actual transfer characteristics are only linear at small amplitudes. For increasing amplitudes, the transfer 


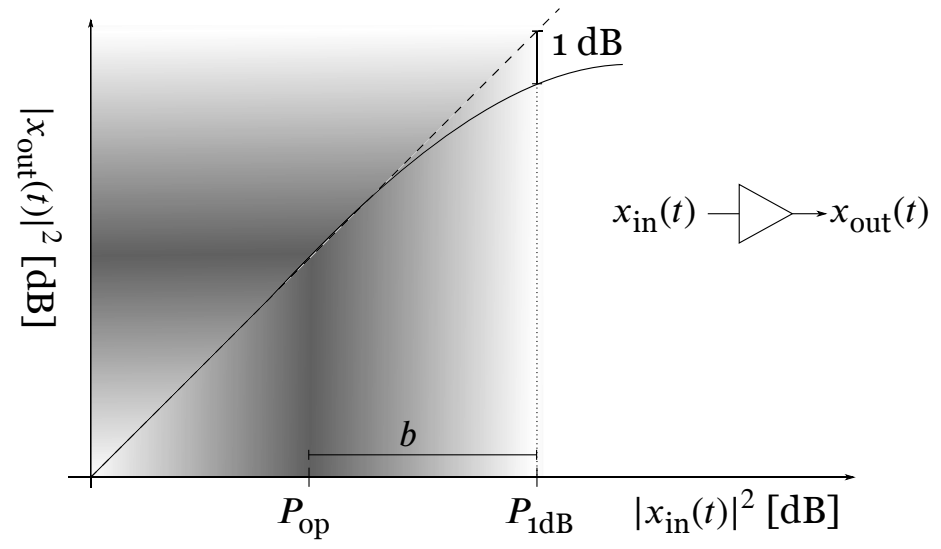

Figure 8: The transfer characteristics of a general power amplifier (solid) compared to a perfectly linear characteristics (dashed).

characteristics saturate, i.e. the output asymptotically approaches a constant. Saturation happens close to the $1-d B$ compression point, which is where the actual transfer characteristics is $1 \mathrm{~dB}$ below the ideal linear one; it is denoted by $P_{1 \mathrm{~dB}}$ in Figure 8 . When an input signal varies inside the nonlinear region, two types of distortion arise: in-band distortion that disturbs the own system and out-of-band radiation that disturbs systems that use adjacent frequency bands. The spectrum of a nonlinearly amplified signal and the out-of-band radiation can be seen in Figure 1 in Paper $\mathrm{C}$.

To avoid distortion in a system without a DPD, the input signal is usually backed off prior to amplification, i.e. its power $P_{\mathrm{op}}$ is chosen such that the amplitude of the input signal most of the time varies within the linear region of the transfer characteristics. The color of the shade in Figure 8 illustrates the time the signal assumes a certain amplitude. The light shade in the nonlinear region indicates that it seldom assumes values there. Through back-off, the shaded area can be moved left and right. The drawback of backoff is a lower power efficiency, which can be seen in Figure 9 for a class B and a Doherty amplifier. The back-off is defined as the fraction between the 1 - $\mathrm{dB}$ compression point and the power of the input signal, $b \triangleq P_{1 \mathrm{~dB}} / P_{\mathrm{op}}$ in Figure 8.

As a first estimate of how much back-off that is needed, the peak-to-average ratio (PAR) is often used. Many times, the PAR is defined as the $\epsilon$-percentile of the complimentary cumulative distribution of the amplitude of the input 


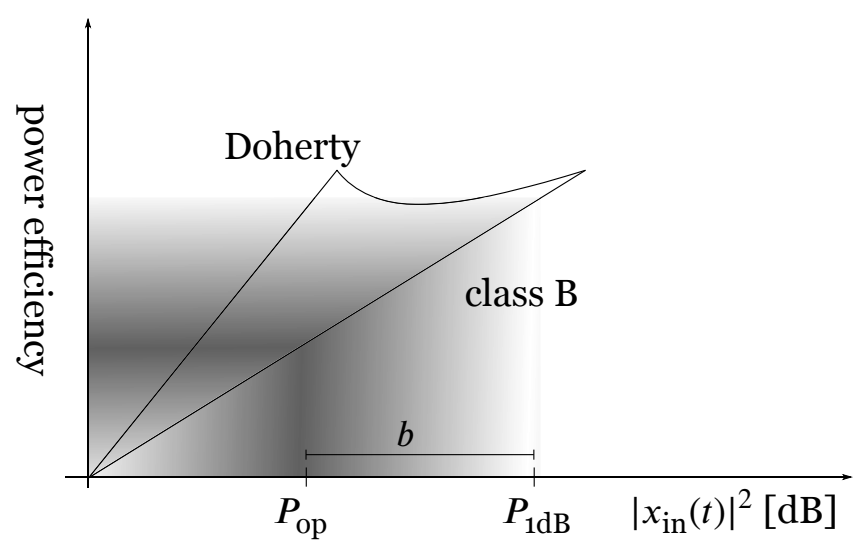

Figure 9: Comparison of the power efficiencies of a class B and a Doherty amplifier as a function of the input power.

signal, i.e.

$$
\mathrm{PAR} \triangleq \sup \left\{A: \operatorname{Pr}\left(\frac{\left|x_{\mathrm{in}}(t)\right|}{\sqrt{\mathrm{E}\left[\left|x_{\mathrm{in}}(t)\right|^{2}\right]}}>A\right)>\epsilon\right\} .
$$

The percentile can be chosen differently depending on the sensitivity of the system to nonlinear amplification. A common choice is $\epsilon=10^{-4}$. The complimentary cumulative amplitude distribution for some massive MIMO signals is illustrated in Figure 1 in Paper A. There it can be seen that with linear precoding the PAR is around $10 \mathrm{~dB}$, independently of modulation order and whether single-carrier or OFDM transmission is used, and with the discretetime constant-envelope precoder, a low-PAR precoding method, the PAR is just over $4 \mathrm{~dB}$.

There are many different ways to model the transfer characteristics of an amplifier [38]. In most models, the baseband equivalent of the output signal $x_{\text {out }}(t)$ is given in terms of the baseband equivalent of the input signal $x_{\text {in }}(t)$. Such models can describe the distortion of the passband signal around the carrier frequency and in the adjacent frequency bands. However, they cannot describe the distortion caused by higher order harmonics, i.e. distortion that can be seen around frequencies that are multiples of the carrier frequency. Since such distortion is far away from the transmission band, however, it can be filtered out relatively easy by the low-order bandpass filter preceding the antenna in Figure 7. A model that neglects the effects of the higher order harmonics is thus still valid in a system with bandpass filters. 
In one amplifier model, the amplified signal is given as follows:

$$
x_{\text {out }}(t)=a\left(\left|x_{\mathrm{in}}(t)\right|\right) e^{j 2 \pi\left(\arg x_{\mathrm{in}}(t)+\Phi\left(\left|x_{\mathrm{in}}(t)\right|\right)\right)} .
$$

The function $a(x)$ is called the $A M-A M$ conversion and describes how the amplitude of the output signal is affected by the amplitude of the input signal. The function $\Phi(x)$ is called the $A M-P M$ conversion and describes how the phase distortion is affected by the input amplitude. There are many ways to model $a(x)$ and $\Phi(x)$, see [39] for a survey. In Paper A, the Rapp model is used, which is given by

$$
\begin{aligned}
a(x) & =A_{\max } \frac{x / x_{\max }}{\left(1+\left(x / x_{\text {max }}\right)^{2 d}\right)^{\frac{1}{2 d}}} \\
\Phi(x) & =0,
\end{aligned}
$$

where $d=2$ was used to model a typical moderate-cost solid-state amplifier [40]. The factors $A_{\max }$ and $x_{\max }$ are parameters that determine the amount of amplification and the point where saturation occurs.

A more general model is given by the Volterra series [41], which is a model on the passband signals:

$$
x_{\text {out }}^{\mathrm{pb}}(t)=b_{0}+\sum_{\varpi=1}^{\Pi} \int_{-\infty}^{\infty} \cdots \int_{-\infty}^{\infty} b_{\varpi}^{\prime}\left(\tau_{1}, \ldots, \tau_{\varpi}\right) \prod_{\varpi^{\prime}=1}^{\varpi} x_{\text {in }}^{\mathrm{pb}}\left(t-\tau_{\varpi^{\prime}}\right) \mathrm{d} \tau_{\varpi^{\prime}} .
$$

where $\Pi$ is the order of the model and $b_{\varpi}^{\prime}\left(\tau_{1}, \ldots, \tau_{\varpi}\right)$ is the kernel of the $\varpi$-dimensional convolution. It has, however, been observed that an amplifier can be accurately described using only the diagonal kernels, i.e. by making the $\varpi$-dimensional convolutions one dimensional:

$$
b_{\varpi}^{\prime}\left(\tau_{1}, \ldots, \tau_{\varpi}\right)=A b_{\varpi}^{\mathrm{pb}}\left(\tau_{1}\right) \prod_{\varpi^{\prime}=2}^{\varpi} \delta\left(\tau_{\varpi^{\prime}}-\tau_{1}\right),
$$

where the impulse response $b_{\varpi}^{\mathrm{pb}}(\tau)$ defines the one-dimensional convolutions and $A$ is a scaling factor. Furthermore, the even-order impulse responses $\left\{b_{\varpi}^{\mathrm{pb}}(\tau), \varpi\right.$ even $\}$ only affect the spectrum of the output signal around even multiples of the carrier frequency, the higher order harmonics. When the distortion around these frequencies are filtered away by the bandpass filter at the antenna, only the odd-order convolutions need to be considered. The polynomial model is obtained by using (98) with

$$
A=\left(\frac{\varpi-1}{2}\right) !\left(\frac{\varpi+1}{2}\right) ! / \varpi !
$$




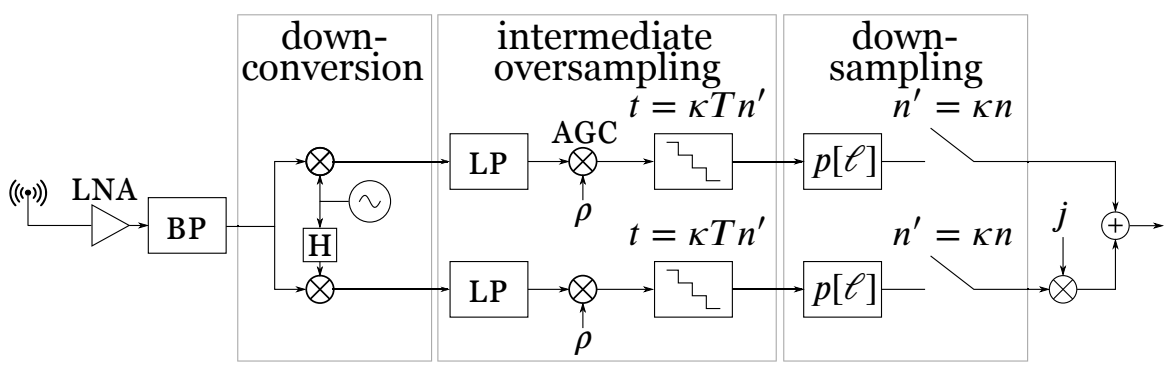

(a) Conventional homodyne design

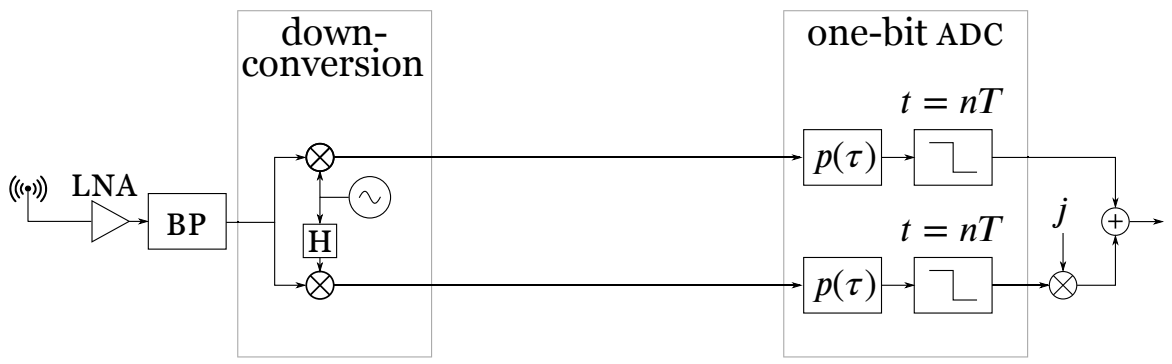

(b) Studied one-bit design

Figure 10: The general receiver design

and only the odd-order kernels to rewrite the Volterra series in (97) to complex baseband as:

$$
x_{\text {out }}(t)=\sum_{\varpi=1,3,5, \ldots, \Pi} \int_{-\infty}^{\infty} b_{\varpi}(t-\tau) x_{\text {in }}(\tau)\left|x_{\text {in }}(\tau)\right|^{\frac{\varpi-1}{2}} \mathrm{~d} \tau,
$$

where the baseband kernel $b_{\varpi}(\tau)$ is related to the passband kernel $b_{\varpi}^{\mathrm{pb}}(\tau)$ as in (9). The kernels $b_{\varpi}(\tau)$ and order $\Pi$ are chosen to fit measured data. The polynomial model is used in Paper $\mathrm{C}$ to analytically obtain an expression for the frequency-dependent covariance matrix of the amplified transmit signals. The polynomial model and other specializations of the Volterra series can be found in [42].

\subsection{Receiver Design}

The receiver design shown in Figure 10(a) is a so called homodyne receiver, where the analog passband signal is downconverted directly to baseband. 


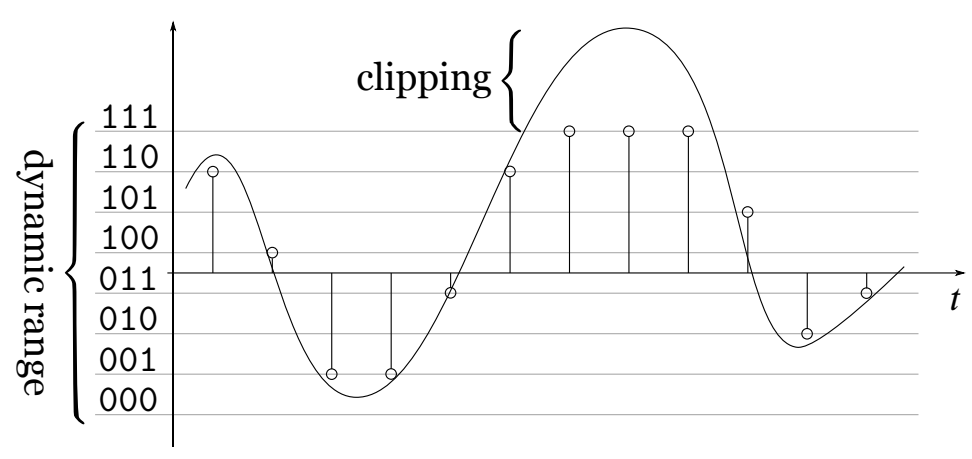

Figure 11: Uniform quantization in a 3-bit ADC

The alternative would be a heterodyne receiver with an intermediate stage, where the analog signal is downconverted to an intermediate low frequency and the baseband signal is recovered in the digital domain.

First, the received signal is amplified by a low-noise amplifier (LNA) and bandpass filtered. To obtain the real and imaginary parts of the baseband signal, the passband signal is split into two copies and downconverted by mixing each copy with their carrier waves, one being a $90^{\circ}$ phase shifted copy of the other. Since this is done in the analog domain in a homodyne receiver, the phase shift might be slightly different from $90^{\circ}$ and the two carrier waves might have slightly different amplitudes. Such imperfections can cause a nonlinear distortion to the complex baseband signal, so called IQ-imbalance. In contrast, in the heterodyne receiver, IQ-imbalance can be avoided at the cost of more complex hardware and higher sampling rate.

The baseband signals are obtained by lowpass filtering the downconverted signals. They are then sampled by an ADC (analog-to-digital converter) with an oversampling factor $\kappa$. The ADC rounds the sample values off to a grid of predetermined quantization levels as shown in Figure 11. The resolution of the ADC is often given in the number of bits required to represent all the quantization levels; an ADC with $Q=8$ levels is thus a $\log _{2} Q=3$-bit ADC.

The dynamic range of the ADC is the range of amplitudes between the lowest quantization level and the highest. If the amplitude of the input signal assumes values outside the dynamic range, the signal is clipped, i.e. its samples are rounded off to the highest or the lowest quantization level depending on which is closer. If, on the other hand, the amplitude of the input signal is much smaller than the dynamic range, not all quantization levels are used and some of the resolution of the ADC is wasted. 
To avoid clipping and to efficiently use the whole resolution, the power of the input signal is controlled by an automatic gain control (AGC), which adjusts the power of the signal such that it most of the time stays just within the dynamic range. Since the power level of the received signal changes over time, the AGC has to be built as a dynamic control loop with both variable attenuators and amplifiers. Because the AGC is a relatively complex technology, a typical base station employs high-resolution ADCs (10-14 bits) to accommodate for a low-end AGC with imperfect power control. The high ADC resolution is also used to handle interference from undesired signals, which otherwise can cause the desired signal to drown and disappear in the quantization.

It is critical that the receive filter $p(\tau)$ in (13) is implemented accurately to avoid improper aliasing in the final symbol-sampled baseband signal. Since it is easier to implement the receive filter as a digital filter, the received signal is usually first sampled by an ADC into an intermediate stage with $\kappa$-times oversampling. After that, it is filtered by a digital receive filter $p[\ell] \triangleq p(\ell T / \kappa)$ and downsampled to the baudrate. In the last stage of the receiver, the two separately recovered real baseband signals are combined into a complex baseband signal.

In massive MIMO receivers, much of the complexity and power consumption stem from the ADCs, whose total complexity scales linearly in the number of antennas. For example, the power consumption and hardware complexity grows exponentially with the number of resolution bits in flash ADCs, which is a type of high-speed ADCs that are used in ultra-wideband systems [43]. Another common ADC design for base stations is the successive approximation ADC, which has lower hardware complexity than flash ADCs and thus can be used to obtain a higher resolution. Successive approximation ADCs, however, are slow and are difficult to use in wideband systems. Especially, at sampling rates above $100 \mathrm{MHz}$, which are necessary if $\kappa / T \geq 100 \mathrm{MHz}$, the power consumption of the ADCs becomes critical, independently of their hardware architecture [44].

Both power consumption and hardware complexity would be reduced by using ADCs with lower resolution. In Paper D, ADCs with the lowest possible resolution (1 bit) and lowest possible sampling rate $(\kappa=1)$ are investigated. To operate the ADC without oversampling $\kappa=1$, i.e. at the baudrate, the analog-to-digital conversion has to be done together with the downsampling without the intermediate stage, as in Figure 10(b). Such a design requires the receive filter to be implemented in the analog domain. Because the 
receive filter, in general, is a steep filter (often a root-raised cosine filter), it would have to be implemented as a high-order active filter, which might be challenging in practice.

If oversampling results in better performance, however, the achievable rates derived in Paper D would lower bound the performance of massive MIMO with low-resolution ADCs. The derived rates should serve as an approximation of the rates that would be achievable in a more practical oversampled system with one-bit ADCs, and give insight into the feasibility of coarse quantization in massive MIMO. It is, for example, observed that it will be difficult for weak users, i.e. users whose received signal is much weaker than the signals from the other users, to obtain good channel estimates, which degrades their performance compared to an unquantized system significantly. To avoid weak users, one-bit ADCs therefore have to be accompanied by user scheduling and proper power allocation.

The use of one-bit ADCs, apart from low power consumption and low hardware complexity, have at least two more advantages. If the one-bit ADCs use a zero threshold, they are insensitive to the absolute magnitude of the amplitudes, only the sign of the signal matters. Therefore the ADC does not require any AGC, which reduces the hardware complexity further. As discussed in Paper D, the active receive filter hypothetically might still need a mechanism to regulate its input voltage to avoid producing nonlinear distortion. Since, in the end, only the sign of the signal is kept in the quantization anyway, nonlinear distortion might not necessarily have to be avoided. In any case, such a mechanism ought to be simpler to implement than the complex AGC required for conventional ADCs.

The second advantage is that the resulting complex baseband signals will be represented with only two bits. This is especially advantageous in a wideband massive MIMO system, where the amount of baseband data that has to be sent between the receiver and the baseband processing unit is huge-it scales linearly with the numbers of antennas, the baudrate and the number of resolution bits. For example, with 100 antennas that each has two conventional 10-bit ADCs, a baudrate of $50 \mathrm{MHz}$ and an oversampling factor of $\kappa=5$, there will be $500 \mathrm{Gbit} / \mathrm{s}$ of data to transfer from all antennas. With an energy consumption of $28 \mathrm{pJ} /$ bit [45], the data transfer will consume $14 \mathrm{~W}$ of power, which might be a significant part of the total hardware power consumption. It is also possible that the large amount of arithmetics that has to be performed on the signals in the baseband processing unit can be simplified by the small number of bits. 


\section{Chapter 4}

\section{Future Work}

It is my intention to continue to develop the research that is presented in this thesis. There are three main directions that should be further investigated:

- continuous-time constant-envelope precoding,

- amplifier distortion from MIMO arrays and

- the use of low-resolution ADCs in massive MIMO.

The continuous-time constant-envelope precoder presented in Paper B fulfills its purpose in that it produces continuous-time signals with $0 \mathrm{~dB}$ PAR and has a mechanism to regulate the bandwidth of the transmit signal. The continued research on this precoder will focus on the possibility to lower its computational complexity and improve upon its performance in terms of increasing the rate of the transmission and lowering the bandwidth of the transmit signal.

The spatial characteristics of the signal distortion caused by nonlinear hardware in the MIMO array should be investigated further. The out-of-band radiation from MIMO arrays was investigated in Paper C. Also the in-band distortion should be analyzed in future work. Furthermore, how different system parameters, such as the characteristics of the propagation environment and the number of antennas and served users, influence the spatial properties of the distortion remains a research topic. 
In the research on ADCs, there are three main topics that should be addressed in future research. The first is to extend the rate analysis in Paper D to general low-resolution ADCs. The second is to study the more practical system setup, where the analog receive signal is sampled and quantized into an intermediate oversampled step and where the receive filter is implemented in the digital domain. The third is to investigate how frequency synchronization can be done in a coarsely quantized system. 


\section{Bibliography}

[1] T. L. Marzetta, "Noncooperative cellular wireless with unlimited numbers of base station antennas," IEEE Transactions on Wireless Communications, vol. 9, no. 11, pp. 3590-360o, Oct. 2010.

[2] F. Boccardi, R. W. Heath, Jr., A. Lozano, T. L. Marzetta, and P. Popovski, "Five disruptive technology directions for $5 \mathrm{G}$," IEEE Communications Magazine, vol. 52, no. 2, pp. 74-80, Feb. 2014.

[3] E. G. Larsson, O. Edfors, F. Tufvesson, and T. L. Marzetta, "Massive MIMO for next generation wireless systems," IEEE Communications Magazine, vol. 52, no. 2, pp. 186-195, Feb. 2014.

[4] X. Gao, F. Tufvesson, O. Edfors, and F. Rusek, "Measured propagation characteristics for very-large MIMO at $2.6 \mathrm{GHz}$," in Proceedings of Asilomar Conference on Signals, Systems and Computers, Nov. 2012, pp. 295-299.

[5] C. Shepard, H. Yu, N. Anand, E. Li, T. Marzetta, R. Yang, and L. Zhong, "Argos: Practical many-antenna base stations," in Proceedings of the 18th Annual International Conference on Mobile Computing and Networking. ACM, Aug. 2012, pp. 53-64.

[6] J. Vieira, S. Malkowsky, K. Nieman, Z. Miers, N. Kundargi, L. Liu, I. Wong, V. Öwall, O. Edfors, and F. Tufvesson, "A flexible 100-antenna testbed for massive MIMO," in Proceedings of IEEE Global Communications Conference, Dec. 2014, pp. 287-293.

[7] P. Harris, S. Zang, A. Nix, M. Beach, S. Armour, and A. Doufexi, "A distributed massive MIMO testbed to assess real-world performance and feasibility," in IEEE Vehicular Technology Conference, May 2015, pp. 1-2. 
[8] E. Björnson, J. Hoydis, M. Kountouris, and M. Debbah, "Massive MIMO systems with non-ideal hardware: Energy efficiency, estimation, and capacity limits," IEEE Transactions on Information Theory, vol. 60, no. 11, pp. 7112-7139, Nov. 2014.

[9] E. Björnson, M. Matthaiou, and M. Debbah, "Massive MIMO with nonideal arbitrary arrays: Hardware scaling laws and circuit-aware design," IEEE Transactions on Wireless Communications, vol. 14, no. 8, pp. 4353-4368, Aug. 2015.

[10] U. Gustavsson, C. Sanchez Perez, T. Eriksson, F. Athley, G. Durisi, P. N. Landin, K. Hausmair, C. Fager, and L. Svensson, "On the impact of hardware impairments on massive MIMO," in Proceedings of IEEE Global Communications Conference, Dec. 2014.

[11] S. Mohammed and E. G. Larsson, "Single-user beamforming in largescale MISO systems with per-antenna constant-envelope constraints: The doughnut channel," IEEE Transactions on Wireless Communications, vol. 11, no. 11, pp. 3992-4005, Sep. 2012.

[12] - - "Per-antenna constant envelope precoding for large multi-user MIMO systems," IEEE Transactions on Communications, vol. 61, no. 3, pp. 1059-1071, Mar. 2013.

[13] - , "Constant-envelope multi-user precoding for frequency-selective massive MIMO systems," IEEE Wireless Communications Letters, vol. 2, no. 5, pp. 547-550, Oct. 2013.

[14] C. Studer and E. G. Larsson, "PAR-aware large-scale multi-user MIMOOFDM downlink," IEEE Journal on Selected Areas in Communications, vol. 31, no. 2, pp. 303-313, Feb. 2013.

[15] C. Risi, D. Persson, and E. G. Larsson, "Massive MIMO with 1-bit ADC," ArXiv E-Print, Apr. 2014, arXiv:1404.7736 [cs.IT].

[16] M. T. Ivrlač and J. A. Nossek, "Challenges in coding for quantized MIMO systems," in Proceedings of IEEE International Symposium on Information Theory, Jul. 2006, pp. 2114-2118.

[17] A. Mezghani and J. A. Nossek, "On ultra-wideband MIMO systems with 1-bit quantized outputs: Performance analysis and input optimization," in Proceedings of IEEE International Symposium on Information Theory, Jun. 2007, pp. 1286-1289. 
[18] J. Mo and R. W. Heath, Jr., "High SNR capacity of millimeter wave MIMO systems with one-bit quantization," in Proceedings of Information Theory and Applications Workshop, Feb. 2014, pp. 1-5.

[19] - - "Capacity analysis of one-bit quantized MIMO systems with transmitter channel state information," IEEE Transactions on Signal Processing, Oct. 2015.

[20] J. Choi, J. Mo, and R. W. Heath, Jr., "Near maximum-likelihood detector and channel estimator for uplink multiuser massive MIMO systems with one-bit ADCs," IEEE Transactions on Communications, vol. 64, no. 5, pp. 2005-2018, Mar. 2016.

[21] A. Lapidoth, A Foundation in Digital Communication. Cambridge University Press, 2009.

[22] T. S. Rappaport, Wireless Communications: Principles and Practice. Prentice Hall, 2002.

[23] J. G. Proakis and M. Salehi, Digital Communications. McGraw-Hill, 2008.

[24] S. Haykin, Communication Systems, 4th ed. Wiley Publishing, 2001.

[25] Q. H. Ngo, E. G. Larsson, and T. L. Marzetta, "Aspects of favorable propagation in massive MIMO," in Proceedings of European Signal Processing Conference, Sep. 2014, pp. 76-80.

[26] E. Björnson, E. G. Larsson, and T. L. Marzetta, "Massive MIMO: Ten myths and one critical question," IEEE Communications Magazine, vol. 54, no. 2, pp. 114-123, Feb. 2016.

[27] J. Jose, A. Ashikhmin, T. L. Marzetta, and S. Vishwanath, "Pilot contamination and precoding in multi-cell TDD systems," IEEE Transactions on Wireless Communications, vol. 10, no. 8, pp. 2640-2651, Aug. 2011.

[28] R. R. Müller, L. Cottatellucci, and M. Vehkaperä, "Blind pilot decontamination," IEEE Journal of Selected Topics in Signal Processing, vol. 8, no. 5, pp. 773-786, Oct. 2014.

[29] M. Médard, "The effect upon channel capacity in wireless communications of perfect and imperfect knowledge of the channel," IEEE Transactions on Information Theory, vol. 46, no. 3, pp. 933-946, May 2000. 
[30] Q. H. Ngo and E. G. Larsson, "No downlink pilots are needed in massive MIMO,” ArXiv E-Print, Jun. 2016, arxiv:1606.02348 [cs.IT.

[31] L. Sanguinetti, E. Björnson, M. Debbah, and A. L. Moustakas, "Optimal linear precoding in multi-user MIMO systems: A large system analysis," in Proceedings of IEEE Global Communications Conference, Dec. 2014, pp. 3922-3927.

[32] H. Yang and T. L. Marzetta, "Performance of conjugate and zero-forcing beamforming in large-scale antenna systems," IEEE Journal on Selected Areas in Communications, vol. 31, no. 2, pp. 172-179, Feb. 2013.

[33] A. Pitarokoilis, S. K. Mohammed, and E. G. Larsson, "On the optimality of single-carrier transmission in large-scale antenna systems," IEEE Wireless Communications Letters, vol. 1, no. 4, pp. 276-279, Apr. 2012.

[34] S. H. Han and J. H. Lee, "An overview of peak-to-average power ratio reduction techniques for multicarrier transmission," IEEE Wireless Communications, vol. 12, no. 2, pp. 56-65, Apr. 2005.

[35] Y. Rahmatallah and S. Mohan, "Peak-to-average power ratio reduction in OFDM systems: A survey and taxonomy," IEEE Communications Surveys and Tutorials, vol. 15, no. 4, pp. 1567-1592, Mar. 2013.

[36] W. H. Doherty, "A new high efficiency power amplifier for modulated waves," Proceedings of the Institute of Radio Engineers, vol. 24, no. 9, pp. 1163-1182, Sep. 1936.

[37] A. Grebennikov, RF and Microwave Power Amplifier Design. McGrawHill, 2005.

[38] J. C. Pedro and S. A. Maas, "A comparative overview of microwave and wireless power-amplifier behavioral modeling approaches," IEEE Transactions on Microwave Theory and Techniques, vol. 53, no. 4, pp. 1150-1163, Apr. 2005.

[39] H. Ochiai, "An analysis of band-limited communication systems from amplifier efficiency and distortion perspective," IEEE Transactions on Communications, vol. 61, no. 4, pp. 1460-1472, Feb. 2013.

[40] N. Benvenuto, R. Dinis, D. Falconer, and S. Tomasin, "Single carrier modulation with nonlinear frequency domain equalization: An idea whose time has come-again," Proceedings of the IEEE, vol. 98, no. 1, pp. 69-96, 2010. 
[41] W. J. Rugh, Linear System Theory. Prentice Hall, 1996, vol. 2.

[42] D. R. Morgan, Z. Ma, J. Kim, M. G. Zierdt, and J. Pastalan, “A generalized memory polynomial model for digital predistortion of RF power amplifiers," IEEE Transactions on Signal Processing, vol. 54, no. 10, pp. 3852-3860, Oct. 2006.

[43] R. H. Walden, "Analog-to-digital converter survey and analysis," IEEE Journal on Selected Areas in Communications, vol. 17, no. 4, pp. 539-550, Apr. 1999.

[44] B. Murmann, “ADC performance survey 1997-2016,” [Online]. Available: http://web.stanford.edu/ murmann/adcsurvey.html, accessed: 2016-07-19.

[45] P. Upadhyaya, J. Savoj, F.-T. An, A. Bekele, A. Jose, B. Xu, D. Wu, D. Furker, H. Aslanzadeh, H. Hedayati et al., "3.3 A 0.5-to-32.75 Gb/s flexible-reach wireline transceiver in $20 \mathrm{~nm}$ CMOS," in IEEE International Solid-State Circuits Conference, Feb. 2015, pp. 1-3. 


\section{Included Papers}





\section{Papers}

The articles associated with this thesis have been removed for copyright reasons. For more details about these see:

$\underline{\text { http://urn.kb.se/resolve?urn=urn:nbn:se:liu:diva-130516 }}$ 



\section{Other Recently Published Theses From \\ The Division of Communication Systems \\ Department of Electrical Engineering (ISY) \\ Linköping University, Sweden}

Antonios Pitarokoilis, Phase Noise and Wideband Transmission in Massive MIMO, Linköping Studies in Science and Technology. Dissertations, No. 1756, 2016.

Anu Kalidas M. Pillai, Signal Reconstruction Algorithms for Time-Interleaved ADCs, Linköping Studies in Science and Technology. Dissertations, No. 1672, 2015.

Ngô Quốc Hiển, Massive MIMO: Fundamentals and System Designs, Linköping Studies in Science and Technology. Dissertations, No. 1642, 2015.

Mirsad Čirkić, Efficient MIMO Detection Methods, Linköping Studies in Science and Technology. Dissertations, No. 1570, 2014.

Reza Moosavi, Improving the Efficiency of Control Signaling in Wireless Multiple Access Systems, Linköping Studies in Science and Technology. Dissertations, No. 1556, 2014.

Johannes Lindblom, The MISO Interference Channel as a Model for Non-Orthogonal Spectrum Sharing, Linköping Studies in Science and Technology. Dissertations, No. 1555, 2014.

Antonios Pitarokoilis, On the Performance of Massive MIMO Systems with Single Carrier Transmission and Phase Noise, Linköping Studies in Science and Technology. Licentiate Thesis, No. 1618, 2013.

Tumula V. K. Chaitanya, HARQ Systems: Resource Allocation, Feedback Error Protection, and Bits-to-Symbol Mappings, Linköping Studies in Science and Technology. Dissertations, No. 1555, 2013. 